ACCEPted to ApJ 29 JAN 2015

Preprint typeset using $\mathrm{LATE}_{\mathrm{E}} \mathrm{X}$ style emulateapj v. 12/16/11

\title{
SPATIALLY EXTENDED Na I D RESONANT EMISSION AND ABSORPTION IN THE GALACTIC WIND OF THE NEARBY INFRARED-LUMINOUS QUASAR F05189-2524
}

\author{
DAvid S. N. Rupke \\ Department of Physics, Rhodes College, Memphis, TN 38112 \\ AND SylVAin VeilleuX \\ Department of Astronomy and Joint Space-Science Institute, University of Maryland, College Park, MD 20742 \\ Accepted to ApJ 29 Jan 2015
}

\begin{abstract}
Emission from metal resonant lines has recently emerged as a potentially powerful probe of the structure of galactic winds at low and high redshift. In this work, we present only the second example of spatially resolved observations of Na I D emission from a galactic wind in a nearby galaxy (and the first 3D observations at any redshift). F05189-2524, a nearby $(z=0.0428)$ ultraluminous infrared galaxy powered by a quasar, was observed with the integral field unit on the Gemini Multi-Object Spectrograph (GMOS) at Gemini South. Na I D absorption in the system traces dusty filaments on the near side of an extended, AGN-driven galactic wind (with projected velocities up to $2000 \mathrm{~km} \mathrm{~s}^{-1}$ ). These filaments $\left(A_{V} \lesssim 4\right.$ and $\left.N(H) \lesssim 10^{22} \mathrm{~cm}^{-2}\right)$ simultaneously obscure the stellar continuum and $\mathrm{Na}$ I D emission lines. The Na I D emission lines serve as a complementary probe of the wind: they are strongest in regions of low foreground obscuration and extend up to the limits of the field of view (galactocentric radii of $3 \mathrm{kpc}$ ). An azimuthally symmetric Sérsic model extincted by the same foreground screen as the stellar continuum reproduces the Na I D emission line surface brightness distribution except in the inner regions of the wind, where some emission-line filling of absorption lines may occur. The presence of detectable Na I D emission in F05189-2524 may be due to its high continuum surface brightness at the rest wavelength of Na I D. These data uniquely constrain current models of cool gas in galactic winds and serve as a benchmark for future observations and models.

Keywords: galaxies: active — galaxies: kinematics and dynamics - galaxies: F05189-2524 - galaxies: ISM — ISM: jets and outflows
\end{abstract}

\section{INTRODUCTION}

Absorption-line probes of galactic winds are now a ubiquitous tool for studying their properties (Veilleux et al. 2005). The suite of metal resonant line transitions in the optical and UV are a particularly powerful tool for finding outflows in the nuclear spectra of galaxies at both low $z$ (e.g., Heckman et al. 2000; Rupke et al. 2002, 2005b c, Martin 2005) and high $z$ (e.g., Shapley et al. 2003 Weiner et al. 2009; Rubin et al. 2010; Martin et al. 2012). Spatially-resolved observations of these lines have recently enabled better constraints on the properties of the cool gas phase of galactic winds in nearby systems (Shih \& Rupke 2010; Rupke \& Veilleux 2011, 2013b).

Redshifted emission from these same transitions has been found in stacked spectra at low and high redshift (Shapley et al. 2003: Weiner et al. 2009: Rubin et al. 2010. Chen et al. 2010, Erb et al. 2012; Kornei et al. 2013), as well as in a handful of individual spectra (Martin \& Bouché 2009; Rubin et al. 2011; Martin et al. 2013). 'This emission is scattered into the line of sight by the outflow due to the fact that the absorption and re-emission occur at the same wavelength. Resonant line emission from cool gas in galactic winds should be a readily observable feature of these winds in the absence of significant dust or strong collimation (Prochaska et al. 2011). The properties of this emission can be used to constrain the wind density and geometry.

drupke@gmail.com
However, only one case of spatially-resolved resonant emission from a galactic wind has been found in the nearby universe (Phillips 1993), despite the large number of nearby outflows that show resonant absorption. NGC 1808 is a nearby early-type spiral inclined $57^{\circ}$ to the line of sight with long dust filaments extending from the nucleus (Phillips 1993, and references therein). Phillips (1993) discovered that the dusty filaments on the near side of a nuclear, bipolar outflow show blueshifted Na I D absorption at projected galactocentric radii of $\lesssim 2 \mathrm{kpc}$, while redshifted Na I D emission lines emerge behind the disk on the far side of the outflow.

Deep observations of two galactic winds at $z=0.7-0.9$ also show spatially resolved Mg II 2796, $2800 \AA$ A emission at $\sim 10 \mathrm{kpc}$ projected radii (Rubin et al. 2011, Martin et al. 2013). These two systems are blue star forming galaxies that show blueshifted absorption and redshifted emission, similar to NGC 1808 but on larger scales.

The nearby $(z=0.0428)$ galaxy F05189-2524 is an ultraluminous infrared galaxy (ULIRG) with a Seyfert 2 optical spectral type (Veilleux et al. 1999a). It is one of the nearest and brightest ULIRGs, and reveals a hidden broad line region in the near-IR (Veilleux et al. 1999b). Approximately $70 \%$ of the bolometric luminosity of F05189-2524 arises from an AGN (Veilleux et al. 2009), meaning that the AGN in this system exceeds the quasar threshold in luminosity $\left(\sim 10^{45} \mathrm{erg} \mathrm{s}^{-1}\right)$. It has a high-velocity outflow, as seen in neutral, ionized, and molecular gas (Rupke et al. 2005a, Westmoquette et al. 


\section{$\mathrm{F} 435 \mathrm{~W}+\mathrm{F} 814 \mathrm{~W}$}

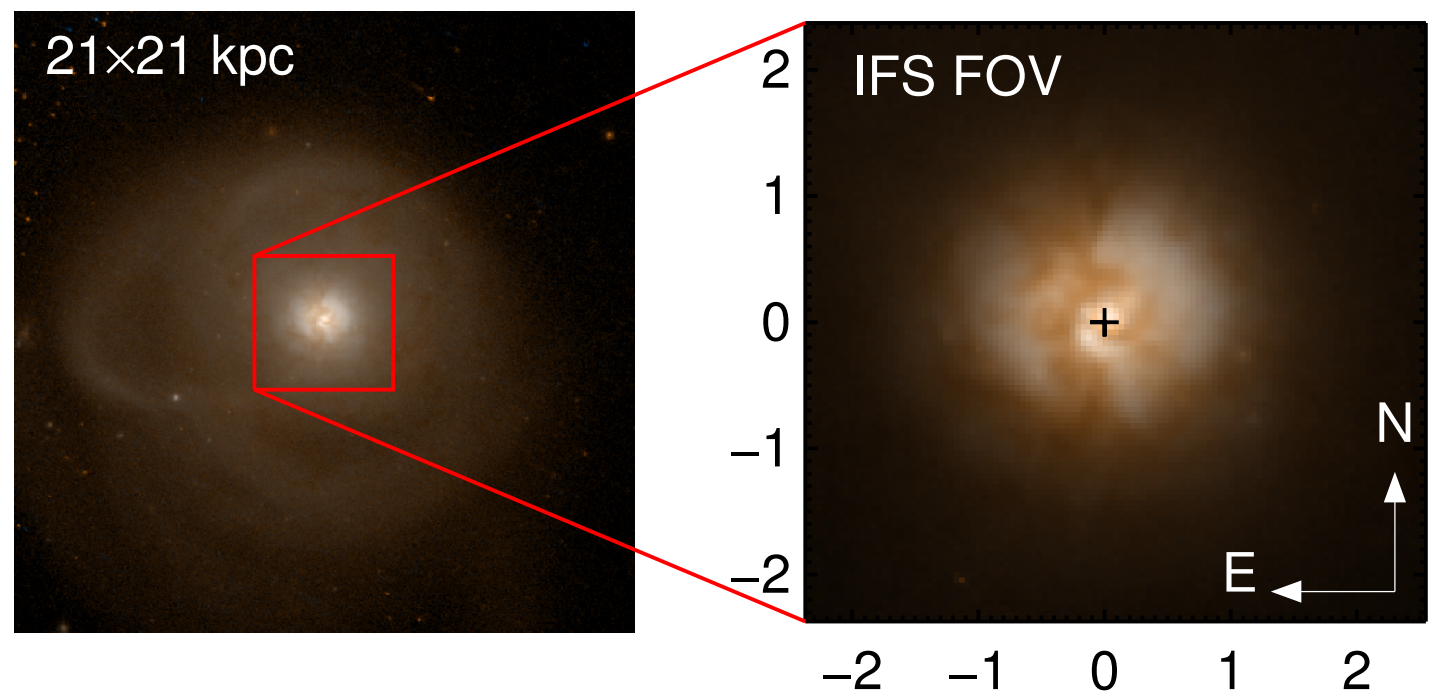

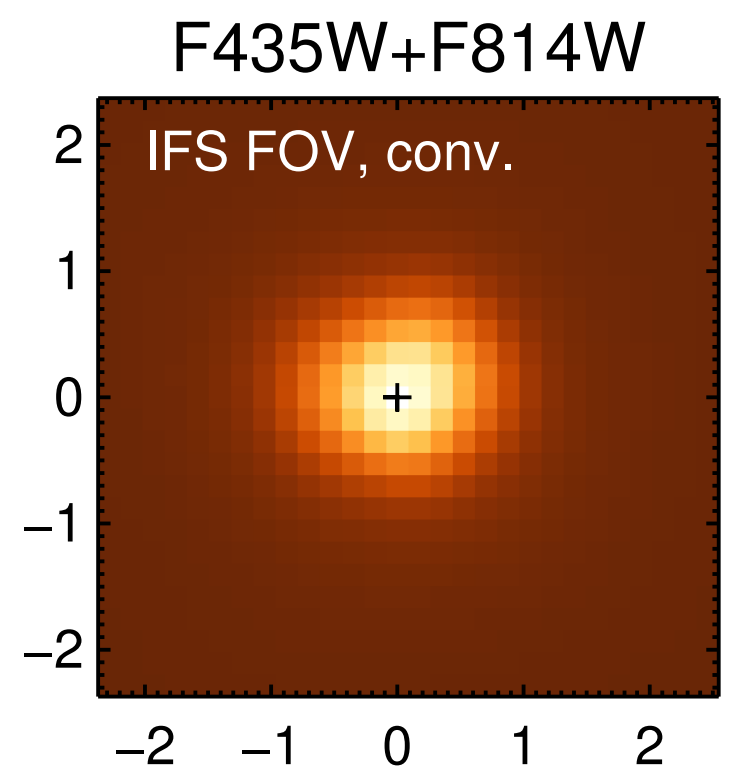

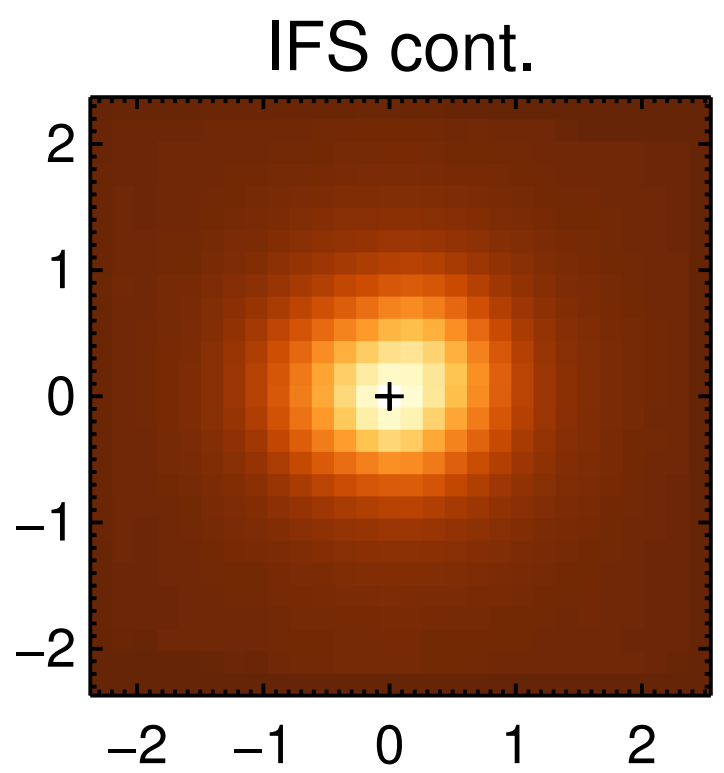

Figure 1. Continuum images of F05189-2524: (top left) HST 3-color image constructed from ACS F435W and F814W exposures, FOV $=25$ " $\times 25$ "; (top right) the same image zoomed into the GMOS FOV (5!' $6 \times 5$ !' 4$)$; (bottom left) the average of the F435W and F814W exposures which is then zoomed, smoothed with a Gaussian kernel of FWHM $=0$ "' 6 to match the ground-based seeing, and rebinned to approximate the IFS data; and (bottom right) the GMOS data, summed between 5600 and $6400 \AA$. The IFS continuum peak is denoted by the cross, and the axes give galactocentric coordinates in kpc. The top panel scaling is asinh (Lupton et al. 1999) with $\beta=0.05$ (left) and $\beta=1$ (right); the lower panel scales are linear.

2012, Veilleux et al.|2013; Teng et al.|2013; Bellocchi et al. 2013). F05189-2524 was observed as part of an integral field spectroscopic survey of nearby major mergers and quasars to study AGN feedback (Rupke \& Veilleux 2011, 2013b; Rupke et al. 2015, in prep.).

Rupke et al. (2005a) reported that this galaxy shows extended $\mathrm{Na} I \mathrm{D}$ emission in long-slit spectra. Here we present integral field observations that show the distribution of resonant line emission and absorption in this system. This is only the second known example of such emission in the local universe, and as such serves as a benchmark for models and observations, both past and future.

In Section 2 we present the observations and our methods of data reduction and analysis. We discuss the equivalent width, Na I D emission line flux, and velocity measurements in Section 3. We connect the dust obscuration of the stellar continuum to both the column of neutral $\mathrm{Na}$ and the emission line flux, and introduce possible wind structures to explain the $\mathrm{Na} I \mathrm{D}$ emission. In Section 4 we outline our preferred model for the $\mathrm{Na}$ I D emission and discuss emission line filling, as well as con- 


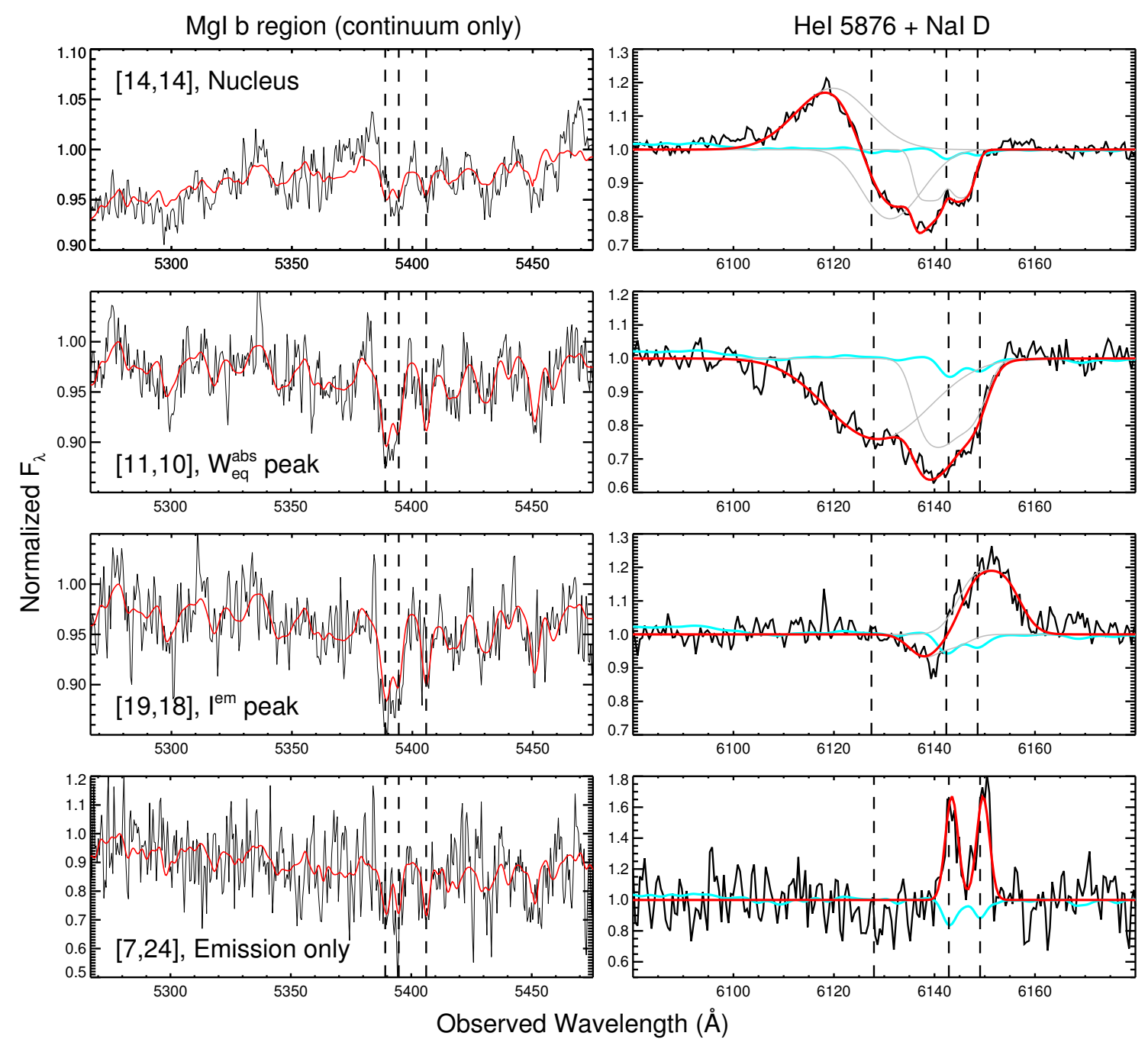

Figure 2. Examples of fits. (Left) Continuum fits to the Mg I b region. The narrow [N I] 5198, $5200 \AA$ and broad [Fe VII] $5159 \AA$ emission lines have been fit and removed. Data is in solid black and total fits are in red. The wavelengths of the Mg I b triplet as expected from the stellar redshift in each spaxel are shown as vertical dashed lines. Each panel is labeled with the spaxel column and row number and any relevant Na I D properties. The top panel fit is affected by residuals from strong and broad [Fe VII] emission. (Right) Fits to Na I D and He I $5876 \AA$. The data is in solid black, the fits to individual components are in gray, the total fits are in red, and the expected wavelengths of Na I D and He I $5876 \AA$ are shown as vertical dashed lines. The underlying stellar continuum by which the data was normalized is shown as solid cyan; the correction for stellar Na I D absorption is evidently small.

sidering the detectability of $\mathrm{Na}$ I D emission in galactic winds. We summarize in Section 5. Throughout the paper we assume $H_{0}=73 \mathrm{~km} \mathrm{~s}^{-1} \mathrm{Mpc}^{-1}, \Omega_{m}=0.27$, and $\Omega_{\Lambda}=0.73$, yielding $0.88 \mathrm{kpc} \operatorname{arcsec}^{-1}$ at the redshift of F05189-2524 $(z=0.0428)$.

\section{OBSERVATIONS, REDUCTION, AND ANALYSIS}

F05189-2524 was observed with the Integral Field Unit in the Gemini Multi-Object Spectrograph (GMOS; Allington-Smith et al. 2002, Hook et al. 2004) on the Gemini South telescope. The integral field unit was used in 1-slit mode with the B600 grating, yielding wavelength coverage from 4600 to $7400 \AA$ and a spectral resolution of $1.6 \AA$ at $6000 \AA$. The Gemini IRAF package (v1.12) and IFSRED ${ }^{1}$ (Rupke 2014b) was employed to reduce the data. Rupke \& Veilleux 2013b present the details of the data reduction. The sky was subtracted using the

\footnotetext{
1 http://github.com/drupke/ifsred
}

average sky spectrum from the 250 spaxels offset $1^{\prime}$ from the field of view (FOV). The standard deviation in the sky flux across the FOV was $<4 \%$ and the median sky fluxes in the sky and science spaxels differed by $<4 \%$, as determined from fits to the [O I] $5577 \AA$ sky line. Seven dithered 30-minute science exposures were combined to yield a FOV of 5 .' $6 \times 5$ !. 4 , and the data was resampled to square spaxels of side 0.22 . The median seeing was $0^{\prime \prime} 6$.

Figure 1 shows Hubble Space Telescope (HST) Advanced Camera for Surveys (ACS) images constructed from F435W and F814W exposures (Armus et al. 2009. Kim et al. 2013). It also presents the IFS continuum integrated between 5600 and $6400 \AA$, compared to a smoothed and rebinned average of the F435W and F814W images.

The continuum and emission lines in each spaxel were modeled using IFSFIT ${ }^{2}$ (Rupke 2014a). In brief, IFS-

\footnotetext{
2 http://github.com/drupke/ifsfit
} 
FIT masks emission line regions, fits the continuum using PPXF (Cappellari \& Emsellem 2004), and then simultaneously fits all emission lines in the continuumsubtracted spectrum. PPXF yields an accurate stellar redshift in each spaxel based on the entire continuum. The range $4600-7100 \AA$ was fit, which contained the absorption and emission lines of interest. A linear combination of stellar templates from González Delgado et al. (2005) and additive Legendre polynomials up to fourth degree yielded a good fit to the continuum of F05189-2524. Though the continuum fit parameters may not be unique due to degeneracies in the continuum models, the fitting nevertheless does an excellent job of reproducing the stellar absorption lines in the continuum and the continuum shape, which is its sole purpose. Figure 2 illustrates the quality of the fits using the continuum region near $\mathrm{Mg} \mathrm{I} \mathrm{b}$, since the strengths of stellar Mg I b and stellar Na I D are proportional (see Rupke et al. 2002, and references therein). The fits are of high quality even in regions strongly affected by emission line residuals (the top left panel of Figure 2, which has had a strong, broad [Fe VII] $5159 \AA$ line removed) or that are noisy (the bottom left panel).

Before modeling the emission lines, the stellar+polynomial continuum was subtracted. Emission lines were modeled with 1-3 Gaussian velocity components. Further details of the emission-line fits for this object will be discussed in a forthcoming paper on outflows in a sample of quasars (Rupke et al. 2015, in prep.).

The Na I D doublet in this galaxy shows absorption, emission, or both across the entire FOV. An empirical and a fit-based approach were employed to measure the properties of the lines. Prior to modeling $\mathrm{Na} I \mathrm{D}$, the spectrum was divided by the continuum fit. This removed the stellar contribution to $\mathrm{Na}$ I D, which has a median and standard deviation of $W_{e q}=0.9 \pm 0.6 \AA$ based on our fits. This stellar contribution is much smaller than the interstellar contribution except at the lowest measured equivalent widths. At these levels the interstellar line is typically strongly blue- or redshifted from the stellar line (§3.2). A second-order multiplicative polynomial was then fit to the region surrounding the $\mathrm{Na}$ I D- He I $5876 \AA$ complex to account for any local systematic errors in the continuum fitting. In Figure 2 we plot both the normalized data and the continuum fit near $\mathrm{Na}$ I D to illustrate that the normalization and removal of the stellar absorption lines change the spectra minimally, and accordingly account for negligible error in the $\mathrm{Na} I \mathrm{D}$ fits.

Example spectra illustrating the range of fit types (described below) are shown in Figure 2. Errors in fit parameters and equivalent widths were determined using Monte Carlo methods (Rupke et al. 2005b).

For the empirical method, the data (signal) and error (noise) spectra were boxcar smoothed using a kernel with FWHM equal to the spectral resolution (Cooksey et al. 2008). Regions where $\mid$ signal/noise $\mid>1.6$ were identified as absorption or emission line detections; the threshold was determined by visual examination of the detection boundaries. The resulting maps of equivalent widths, signal-to-noise ratios (SNRs), and emissionline surface brightnesses (all from direct integration) are shown in Figure 3 . These values all refer to the combined measurements of both doublet lines.

The fitting method required careful treatment of the emission and absorption lines. The emission lines were assumed to each consist of a single, Gaussian velocity component. The flux ratio in the doublet lines can vary between the optically thick $\left(I_{5890}^{e m} / I_{5896}^{e m}=1\right)$ and thin limits $\left(I_{5890}^{e m} / I_{5896}^{e m}=2.01\right.$, equal to the optical depth ratio of the two lines). However, the line ratio is difficult to independently constrain in many spaxels. In spaxels with the highest SNR, the lines are broad and fits with a given line ratio are statistically indistinguishable from others due to the close separation of the two lines $\left(304 \mathrm{~km} \mathrm{~s}^{-1}\right)$. In spaxels with low-to-moderate SNR and adjacent absorption, the chance of absorption mixing with line emission could lead to an underestimate of the flux ratio, since the mixing would preferentially lower the SNR of the $5890 \AA$ line (see $\S 4.1$ for more discussion of emission line filling).

The best spaxels for determining the intrinsic line flux ratio are therefore those with narrow line widths and pure emission. In the current data, these regions occur primarily in the northeast and northwest regions of the FOV. In the NE and NW, two regions were identified, each of $25-26$ adjacent spaxels which had wellconstrained emission line ratios. The median best-fit $\sigma$ in these spaxels is $62 \mathrm{~km} \mathrm{~s}^{-1}$, and the median emissionline SNR of the equivalent width is 10.5. The median best-fit line ratio is 1.00 , and Monte Carlo simulations of the errors yielded a median $68 \%(95 \%)$ confidence level of $I_{5890}^{e m} / I_{5896}^{e m}<1.14(1.55)$. Based on these results, the flux ratio for all other spaxels was fixed to the optically thick case $\left(I_{5890}^{e m} / I_{5896}^{e m}=1\right)$.

The absorption line fitting followed Rupke et al. (2005b), which assumes a Gaussian in optical depth and allows for a non-zero (but constant) covering factor in each velocity component. The absorption lines in F05189-2524 clearly require two velocity components in most of the spaxels with SNR $\gtrsim 15$ (Figure 4). In spaxels with lower SNR, two components did not yield a well-constrained fit, and only one component was used.

The fits tended toward one of two solutions: an optically thick $\left(\tau_{5890} \sim 2\right)$ fit with covering factor $C_{f} \sim 0.2$, and an optically thin $\left(\tau_{5890} \sim 0.1\right)$ fit with $C_{f}=1.0$. The one-component fits (which also had lower SNR) tended to yield lower total optical depths than the two-component fits. We argue in Section 3.1 that the high- $\tau$ fit is generally the correct one.

Accurately measuring the properties of the Na I D absorption on the blue edge of the feature requires accurate modeling of the He I $5876 \AA$ emission line in spaxels where it is present. Emission line fits to He I $6678 \AA$ were used to constrain the properties of He I $5876 \AA$ emission. He I lines in F05189-2524 are broad and blueshifted, and the $6678 \AA$ line was fit with a single Gaussian in the central spaxels (it was too weak at larger galactocentric radii). The central wavelength and line width of the $5876 \AA$ line were fixed to those of $6678 \AA$, while its peak flux was allowed to vary. Detected He I $5876 \AA$ emission is confined to radii of three spaxels or less.

In $\sim 30 \%$ of spaxels containing the $\mathrm{Na} \mathrm{I} \mathrm{D}$ line, both absorption and emission are obviously present. The two are adjacent in wavelength space in these spaxels, with 

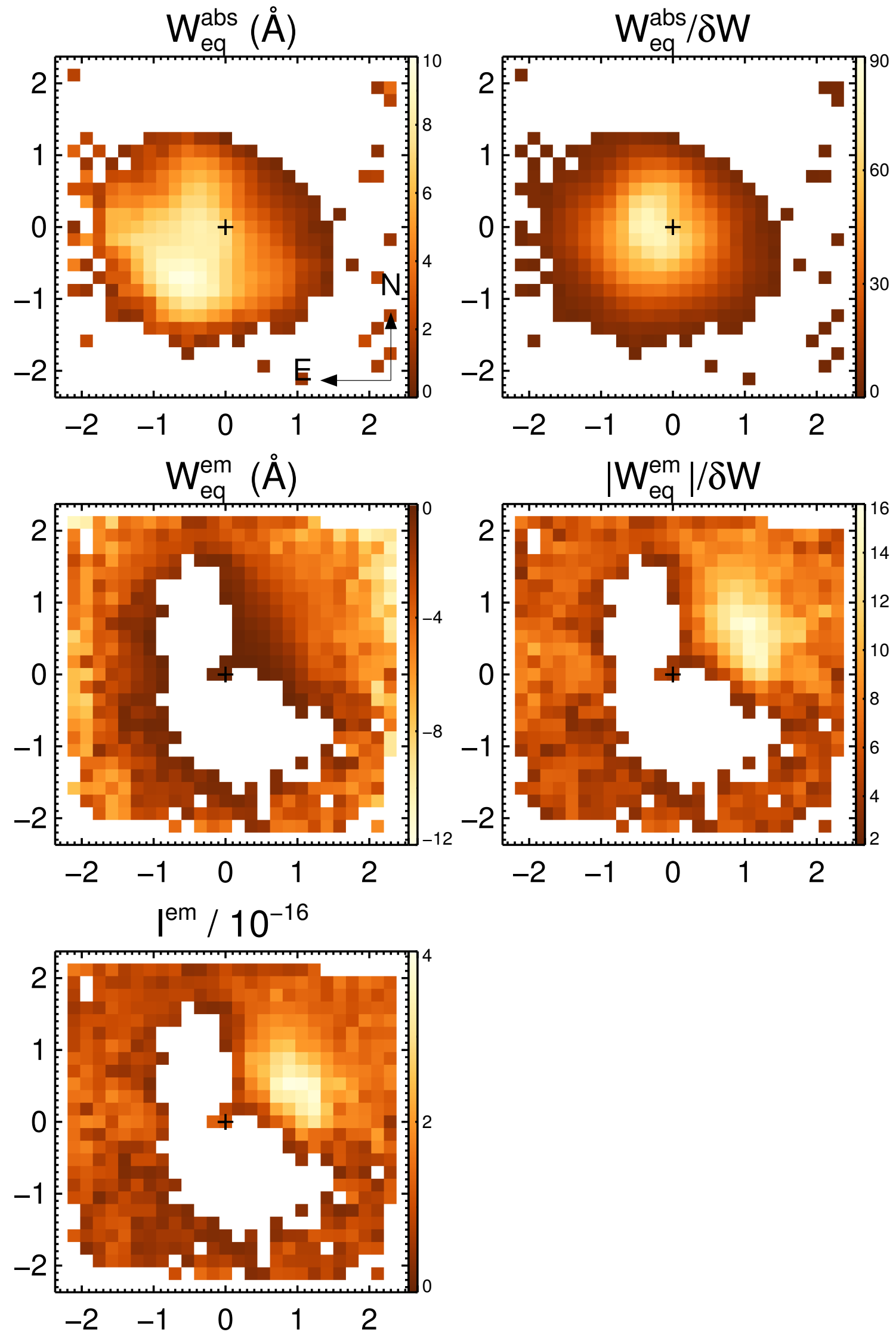

Figure 3. (Top) Observed equivalent width $(\AA)$ of Na I D absorption and its signal-to-noise ratio (SNR). Absorption lines were detected and integrated using an empirical method and corrected for contamination by the nuclear PSF ( $\$ 2$. A $3 \sigma$ threshold was used to mask the data. (Middle) Observed equivalent width $(\AA)$ of Na I D emission and its SNR. (Bottom) Surface brightness of Na I D emission (units of $\left.10^{-16} \mathrm{erg} \mathrm{s}^{-1} \mathrm{~cm}^{-2} \operatorname{arcsec}^{-2}\right)$. The values shown in this figure are the combined measurements of both doublet lines. 
the absorption line blueshifted from the stellar continuum and the emission line near systemic or redshifted. Modeling the absorption and emission lines simultaneously leads to significant degeneracy in the best fit, since increasing the emission line flux and shifting it to the blue can be compensated for in the fit by increasing the absorption line equivalent width and shifting it to the red. To model the Na I D in these spaxels, the emission and absorption lines were thus fit separately. The feature boundaries determined from the empirical method were used to first fit the emission line only using data redward of the absorption line. The emission line properties were then fixed in the absorption line fit.

As a result of this method, it is possible that the emission line equivalent widths are underestimated and that their measured line centers are shifted redward of the true line centers. Correspondingly, the absorption line equivalent widths could be underestimated and the measured line centers shifted blueward. This effect is explored in $\S \S 3.2$ and 4.1 .

The maps of fit-based equivalent widths and emissionline surface brightnesses, as well as comparisons of the two methods, are shown in Figure 5. There is excellent correspondence between the empirical and fitting methods. The fitting method allows better deblending of emission and absorption, and as a result yields slightly higher absorption line equivalent widths (typically $\lesssim 0.5 \AA$, though the difference is higher near the nucleus where He I $5876 \AA$ is strong).

F05189-2524 contains an unresolved nuclear point source due to an AGN (Surace et al. 1998; Kim et al. 2002 Farrah et al. 2005, Veilleux et al. 2006 Kim et al. 2013), which contributes $<1 \%$ of the galaxy's light (Kim et al. 2013). The additive polynomials in our continuum fit may partly trace light from this point source, as the polynomial dominates within a few $\times 0.0^{\prime \prime} 1$ of the nucleus. However, the shape of the intrinsic AGN continuum is unknown and the polynomial-only surface brightness profile is broader than the seeing disk. To more reliably model the spatially unresolved AGN emission, the PSF of the data cube was traced using the bright [Fe VII] $6087 \AA$ line (which is unresolved even at HST resolution; Farrah et al. 2005). The AGN-only continuum emission was then assumed to take the shape of this PSF at the wavelength of $\mathrm{Na}$ I D and to contribute $50 \%$ of the total (or AGN + stellar) continuum surface brightness at the PSF center. The normalization of the AGN contribution is uncertain, but is broadly consistent with the more detailed photometric modeling of Kim et al. (2013) at HST resolution.

Measured equivalent widths were corrected for the contribution of the AGN by dividing $W_{e q}$ by the fractional contribution of the stellar (or total - AGN) continuum to the total $\mathrm{Na}$ I D continuum in each spaxel, as predicted by the PSF model. Low-resolution HST spectra suggest that the nuclear spectrum has little or no $\mathrm{Na}$ I D absorption present (Farrah et al.2005), so correcting for the AGN only affects the continuum level and not the $\mathrm{Na}$ I D line shape. The resulting correction enhanced the nuclear values of the absorption line equivalent width. Significant PSF corrections were confined to the inner few spaxels of the absorption line maps, and have a negligible quantitative impact on the results inferred from these maps.

\section{RESULTS}

This section presents physical quantities inferred from the $\mathrm{Na}$ I $\mathrm{D}$ fits, including gas column density and velocity. It also quantifies the connection of the absorbing and emitting gas to optical obscuration and compares the emission line velocities to other profile properties. The mass outflow rate and energetics of the wind will be presented in a future paper in the context of a larger sample of quasars with outflows (Rupke et al. 2015, in prep.).

\subsection{Dusty Absorption and Obscured Emission}

Correlations exist between cool gas absorption and dust attenuation in nuclear spectra of galaxies (Veilleux et al. 1995; Heckman et al. 2000) as well as within individual galaxies (Shih \& Rupke 2010, Rupke \& Veilleux 2013b), tracking the correlations seen in interstellar sightlines in the Milky Way (Hobbs 1974, Munari \& Zwitter 1997; Vergely et al. 2010). Filametary dust structures in fact cover the nuclear region of F05189-2524 in optical images, causing variation in the emergent optical continuum colors. Figure 6 shows HST ACS $(B-I)$ color maps constructed from $\mathrm{F} 814 \mathrm{~W}$ and F435W exposures (see Surace et al. 1998 for similar WFPC2 color maps). The HST data that is convolved to the ground-based spatial resolution and rebinned to match the GMOS spaxel locations and sizes show a range in $F 435 W-F 814 W$ (AB) colors of $0.8-1.8$. The higher resolution data show a slightly larger range, of $0.6-2.5$, with the upper limit occurring near the nucleus and the lower limit in the blue region $1 \mathrm{kpc} \mathrm{W}$ of the nucleus.

The intrinsic optical spectrum of F05189-2524 is likely dominated by a young stellar population. The presence of a large, concentrated molecular gas reservoir in F05189-2524 (Sanders et al. 1991) and a quasar-like luminosity dominated by reprocessed emission (U et al. 2012 ) indirectly indicate dust-enshrouded, ongoing star formation (Armus et al. 2007; Veilleux et al. 2009). However, the stellar populations that dominate the optical spectrum of F05189-2524 have been constrained only with broadband photometric data (Surace \& Sanders 2000), leaving degeneracies related to the presence of dust, metallicity, and stellar population history (e.g., Calzetti 2001). The metallicity of starburst-dominated ULIRGs is near solar (Rupke et al. 2008), removing one of these degeneracies. The stellar population modeling used in this work (2) is not comprehensive enough to precisely resolve the remaining degeneracies, despite its ability to accurately remove the stellar continuum. Furthermore, the GMOS spectra have a limited wavelength range, including only two Balmer lines that are heavily mixed with emission. Nonetheless, the stellar population age that dominates the luminosity of most spaxels is of order several 10 s to 100 s of Myr. Comprehensive modeling of large samples of ULIRGs show that this is the typical age that dominates ULIRG optical spectra (Rodríguez Zaurín et al. 2009, 2010).

The intrinsic stellar population in F05189-2524 should also vary little across the FOV. The stellar populations in ULIRGs do not change significantly within the inner 3 kpc radius (Soto \& Martin 2010; Rodríguez Zaurín et al. 2009 ), the region probed by our observations. The ages and normalizations of the stellar populations in fits to 


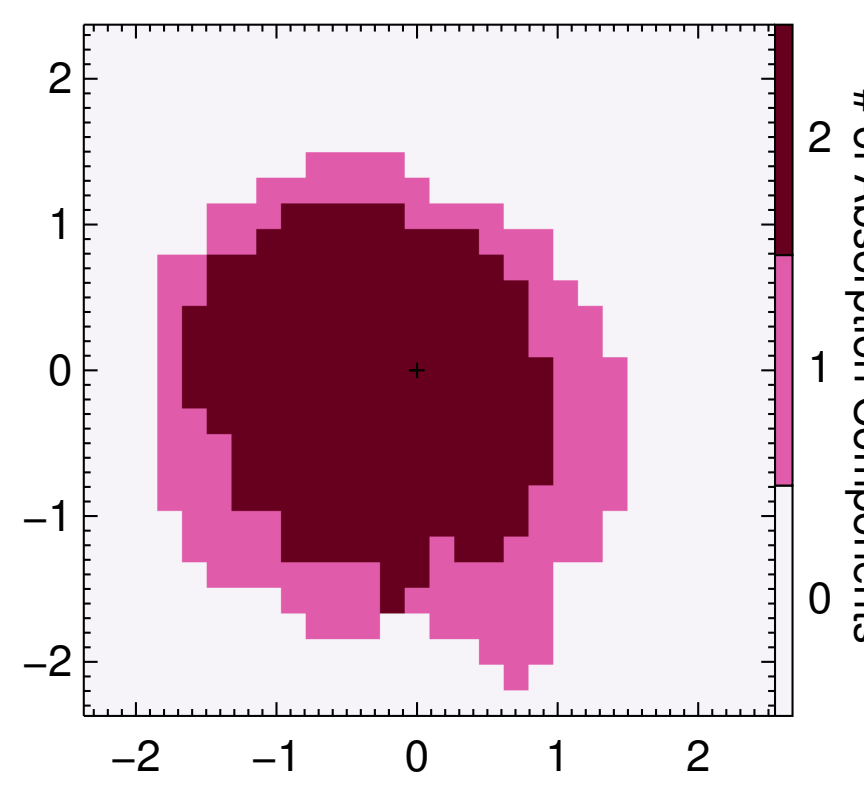

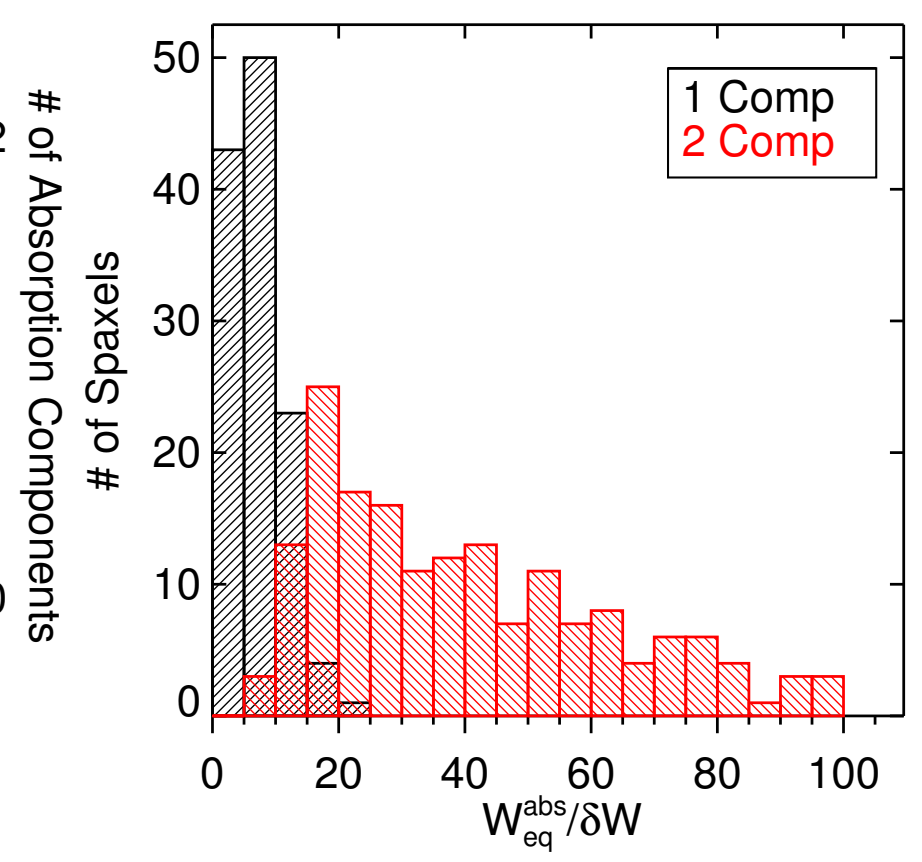

(b)

Figure 4. (Left) Map of number of components in the Na I D absorption line fit. The number of components that can be fit is primarily a function of the SNR, which results in the number of components decreasing with galactocentric distance. (Right) Histograms of absorption line SNR for one-component (black hatched) and two-component (red hatched) fits.

the GMOS data show the same type of population mix across almost the entire FOV. Furthermore, the filamentary structure in the color map (Figure 6) is more consistent with variations in dust column than stellar population.

We thus make the assumption that the $(B-I)$ colors in Figure 6 represent varying levels of dust attenuation rather than significant changes in stellar population. To estimate instrinsic stellar colors, we used Starburst99 (Leitherer et al. 1999) to model the underlying stellar population at optical wavelengths as a solar metallicity continuous burst. Such a burst has $(B-I)_{A B}=0.0$ after $2 \times 10^{7} \mathrm{yr}$. This decreases to $(B-I)_{A B}=-0.3$ at $1 \times 10^{7} \mathrm{yr}$; earlier ages should not dominate the optical spectrum (Rodríguez Zaurín et al. 2009, 2010). These values bracket a small range compared to the observed variation in colors. In our analysis we assume $(B-I)_{A B}=0.0$; lower values will raise the inferred $A_{V}$ by a small amount but otherwise do not affect our analyses.

Using the starburst attenuation curve of Calzetti et al. (2000) with $R_{V}=4.05$, the $H S T$ color ranges then correspond to $A_{V}=1.2-2.8$ (convolved and rebinned) and $0.9-3.9$ (unsmoothed), which are typical of ULIRG nuclei (Veilleux et al. 1999a). These $A_{V}$ correspond to optical depths of $1-4$ at the rest wavelength of $\mathrm{Na}$ I D. Figure 7 therefore shows that optical continuum attenuation is strongly anti-correlated with $\mathrm{Na}$ I D emission line surface brightness $\left(I^{e m}\right)$ and positively correlated with $\mathrm{Na}$ I D absorption line equivalent width $\left(W_{e q}^{a b s}\right)$. It has no correlation with emission line equivalent width $\left(W_{e q}^{e m}\right)$.

However, $W_{e q}^{a b s}$ is an empirical quantity that does not have a one-to-one relationship with column density, particularly in the presence of partial covering of the absorbing gas. The analytic fits to Na I D absorption accurately separate covering factor and optical depth and allow the calculation of hydrogen column density (Rupke et al. 2005b).

The ionization and dust conditions needed to compute $N(H)$ are assumed to be similar to sightlines through the Milky Way (Rupke et al. 2005b): ionization fraction $y \equiv 1-N(\mathrm{Na} \mathrm{I}) / N(\mathrm{Na})=0.9$ and dust depletion of -0.95 dex. The applicability of these values to the potentially more extreme conditions of ULIRGs is uncertain. However, a direct, independent check on $N(H)$ in the outflow comes from the H I absorption measurements of Teng et al. (2013), who find two outflowing components at the same velocities detected in $\mathrm{Na} I \mathrm{D}(\S 3.2)$. For these components, $N(H)=10^{22} \mathrm{~cm}^{-2}$, which is the same as the typical column inferred from $\mathrm{Na} I \mathrm{D}$ in the dustiest regions of this galaxy (Figure 8). Indirect support for the validity of these physical conditions comes from the fact that mass outflow rates calculated using such conditions are comparable to the star formation rate (Rupke et al. 2005c, Rupke \& Veilleux 2013b) and are in agreement with estimates from other cool gas phases (e.g., molecular; Feruglio et al. 2010; Sturm et al. 2011).

The column computed from Na I D can also be compared to the dust column. If the varying $(B-I)$ colors represent dust attenuation, the column density of hydrogen that is along the line of sight in each spaxel (and is associated with the continuum-absorbing dust) can be inferred by applying a dust-to-gas ratio to the attenuation calculated from the stellar color. Figure 8 shows that, within the measurement uncertainties, the two-component fits do a remarkable job of matching 

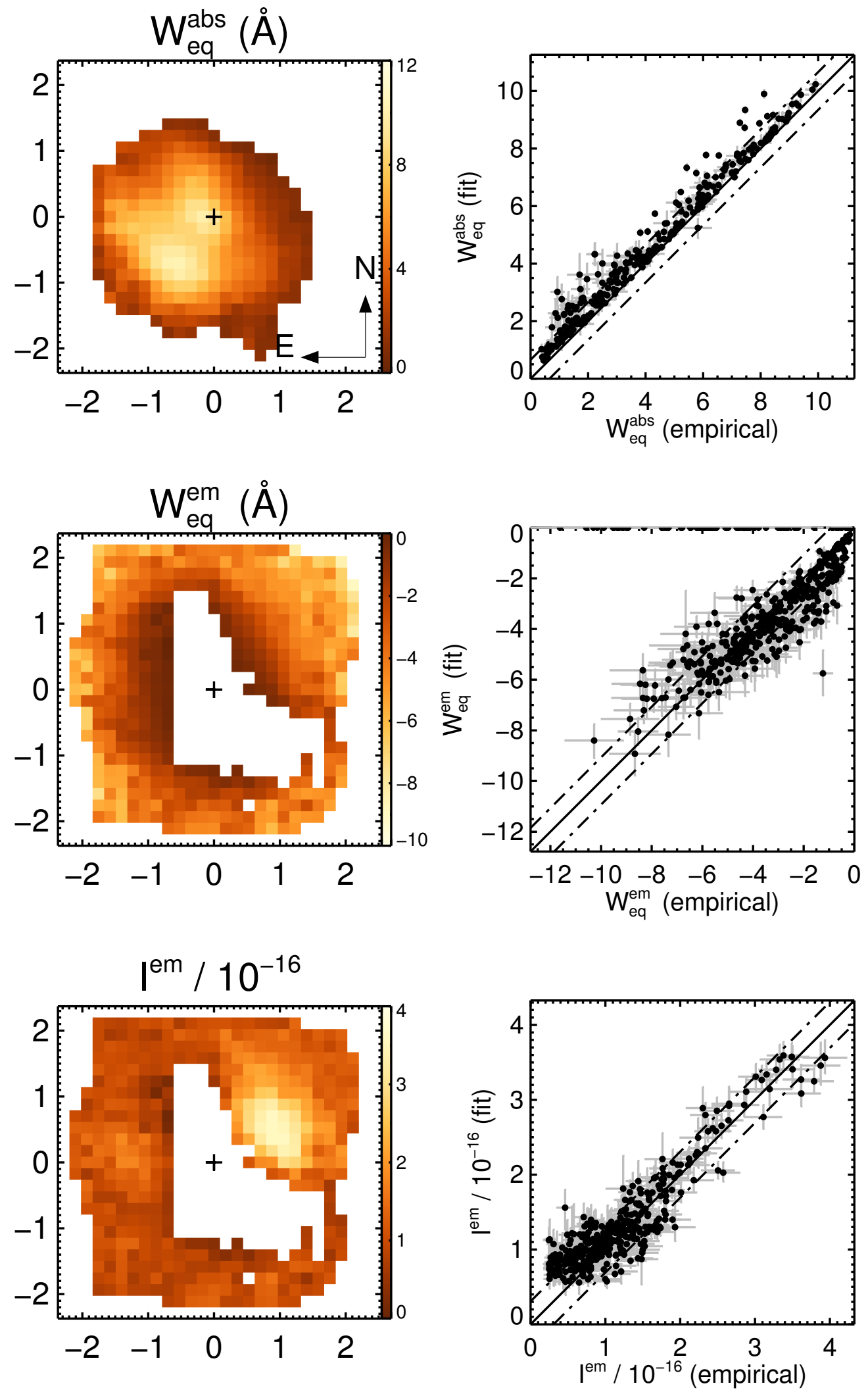

Figure 5. (Top) Observed equivalent width $(\AA)$ of Na I D absorption from fitting with analytic profiles ( 2 , and a comparison of empirical and fit-based equivalent widths. The solid line is equality, and the dot-dashed lines represent the RMS scatter. (Middle) Na I D emission line equivalent width $(\AA)$ and comparison of methods. (Bottom) Na I D emission line surface brightness (units of $10^{-16} \mathrm{erg} \mathrm{s}^{-1} \mathrm{~cm}^{-2}$ $\left.\operatorname{arcsec}^{-2}\right)$ and comparison of methods. The values shown in this figure are the combined measurements of both doublet lines. 


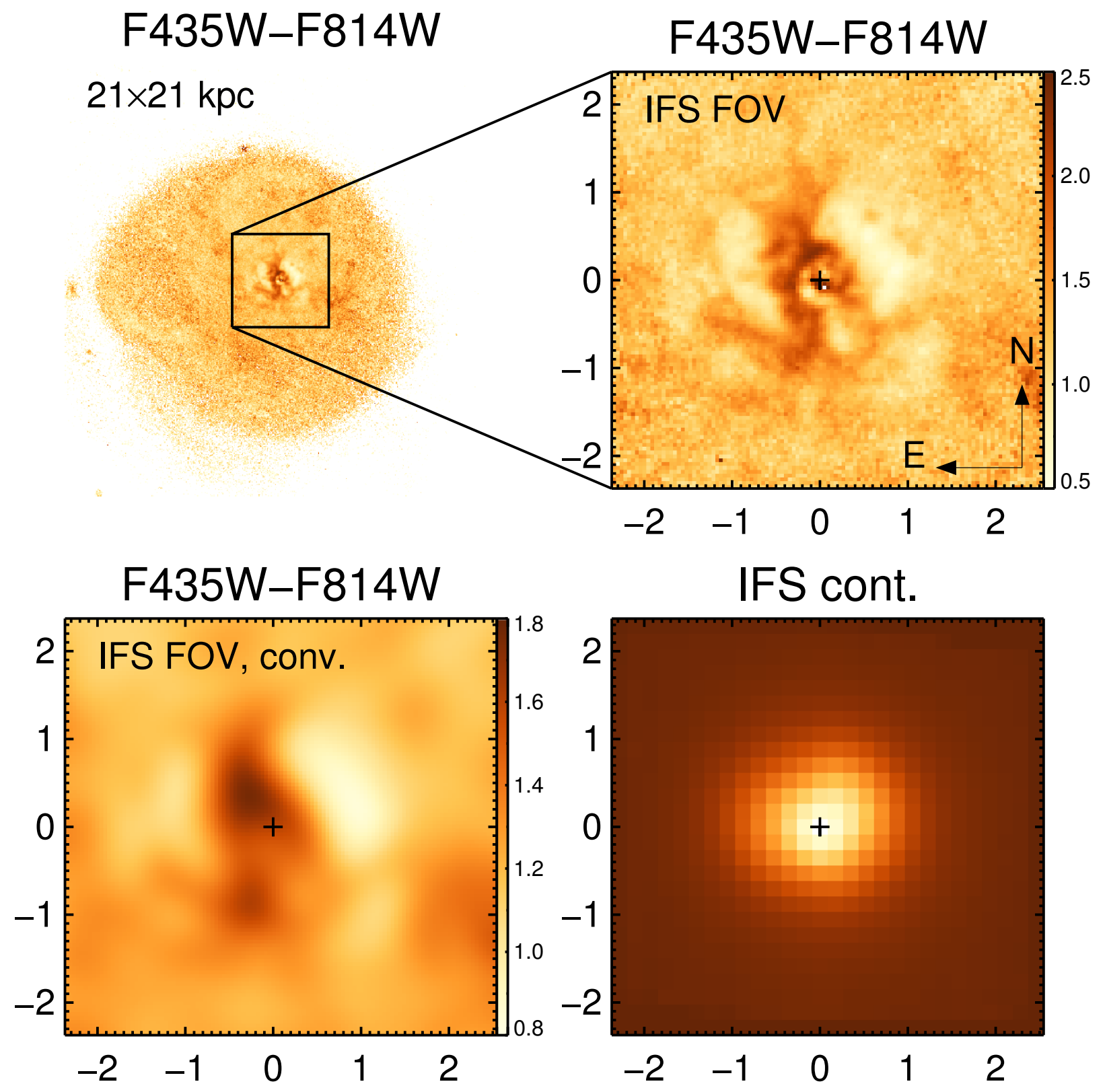

Figure 6. Color images of F05189-2524: (top left) $(B-I)_{A B}$ color map constructed from $\mathrm{F} 435 \mathrm{~W}$ and $\mathrm{F} 814 \mathrm{~W}$ exposures, FOV $=$ 25 " $\times 25$ "; (top right) the same image zoomed into the GMOS FOV (5.' $6 \times 5$ ". 4$)$; (bottom left) the same image, zoomed and smoothed with a Gaussian kernel of FWHM $=0$ "' 6 ; and (bottom right) the GMOS data, summed between 5600 and $6400 \AA$. 

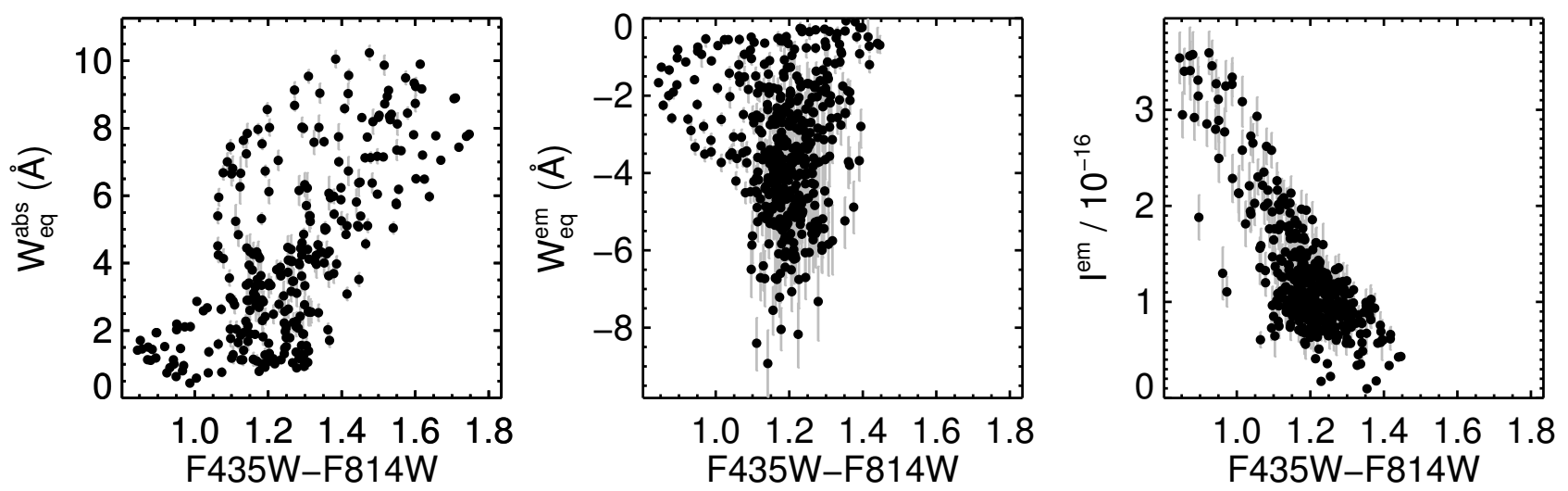

Figure 7. Observed equivalent width $(\AA)$ of Na I D absorption, equivalent width $(\AA)$ of Na I D emission, and surface brightness of $\mathrm{Na}$ I D emission (units of $10^{-16} \mathrm{erg} \mathrm{s}^{-1} \mathrm{~cm}^{-2} \operatorname{arcsec}^{-2}$ ) vs. F435W-F814W (AB) color. The HST continuum data was convolved to the ground-based spatial resolution and rebinned to match the GMOS spaxel locations and sizes. The apparent correlation between $W_{e q}^{a b s}$ and color suggests a connection between dust and cool, neutral gas. The correlation between $I^{e m}$ and color (but not $W_{e q}^{e m}$ and color) suggests that dust regulates the flux $(\S 3.1$.

the dusty gas column for a Galactic dust-to-gas ratio of $N(H) / A_{V}=1.8 \times 10^{21} \mathrm{~cm}^{-2}$ (Predehl \& Schmitt 1995). The one-component fits, on the other hand, for the most part fall below the column density expected from the observed color by factors of $\gtrsim 10$. These fits are primarily in the low-SNR outskirts of the absorbing region (Figure 4 , and favor a $\tau_{5890} \sim 0.1, C_{f} \sim 1.0$ fit to $\mathrm{Na} \mathrm{I} \mathrm{D} \mathrm{vs.}$ the $\tau_{5890} \sim 2, C_{f} \sim 0.2$ fit that emerges with better SNR and two components. The observed emission line optical

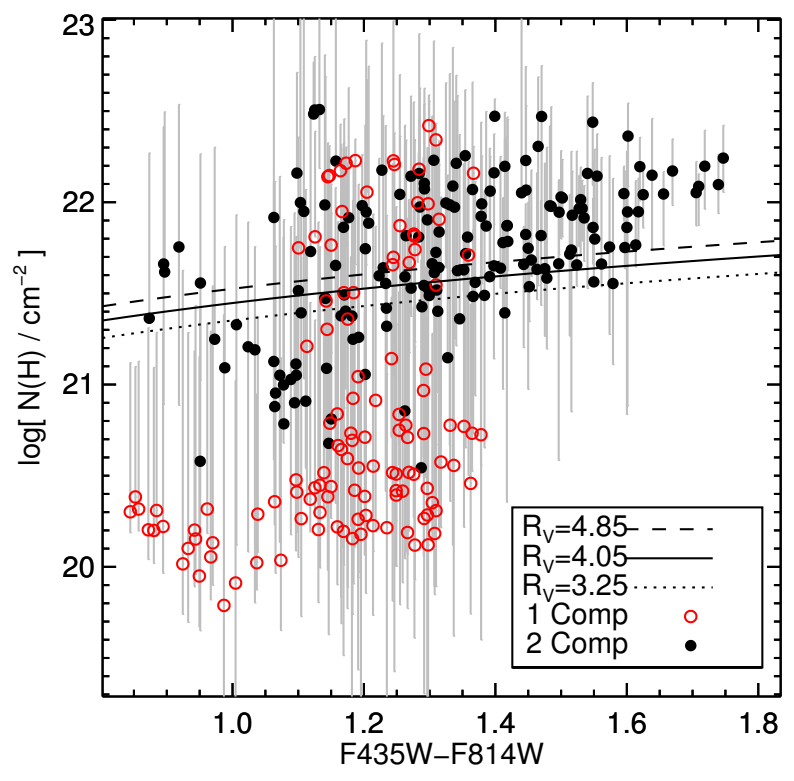

Figure 8. Column density of hydrogen vs. F435W-F814W color. $N(H)$ is computed from Na I D absorption $(\S 2$. The black solid (red open) points represent two (one) component fits. The lines assume that the stellar color primarily reflects dust attenuation, and this fact is used to estimate $N(H)$ from the color given a plausible stellar population, a starburst attenuation curve (Calzetti et al. 2000), and a Galactic dust-to-gas ratio (Predehl \& Schmitt 1995) (\$ 4.1). The discrepancy between one and two component fits reflects the inability of the one component, lower SNR spaxels to simultaneously constrain the covering factor and optical depth. depths in these same regions $(\S 2$ are also consistent with the optically-thick solution. The logical inference is that the one-component fits underestimate the true column density in the absorbing gas, and that higher SNR at these locations would yield the correct optical depth and covering factor $\left(\tau_{5890} \sim 2\right.$ and $\left.C_{f} \sim 0.2\right)$. However, emission line filling could also affect the fits in these spaxels (§4.1).

This physical correspondence between the column densities of neutral, outflowing gas and optical continuum attenuation has several possible consequences, all of which can be inferred from the current data but which need further confirmation with other data to be more generally applicable. (1) The apparent $W_{e q}^{a b s}$-color correlation in the absorbing gas is deceptive (Figure 7). The physical correlation observed in the two-component fits in Figure 8 is more fundamental and less obvious. (2) Correlations between $W_{e q}^{a b s}$ and galaxy color have been previously used to argue that the neutral, outflowing gas probed by $\mathrm{Na}$ I D in dusty active galaxies is also dusty (Veilleux et al. 1995; Heckman et al. 2000; Shih \& Rupke 2010, Rupke \& Veilleux 2013b). However, these conclusions need to be revisited and better quantified using high SNR measurements of Na I D. (3) Accurate measurements of $N(H)$ in these galaxies requires $\mathrm{SNR} \gtrsim 15$ in the equivalent width (Figure 4). (4) The Na I D column densities reported from fits to high SNR nuclear spectra are reasonably accurate (e.g., Heckman et al. 2000, Rupke et al. 2005c a. Rupke \& Veilleux 2011, 2013b). This implies furthermore that the Galactic conditions used to calculate $N(H)$ (Rupke et al. 2005b) are close to the conditions in the cool, foreground gas of dusty outflows in active galaxies. (5) The filamentary continuum structures observed in optical $H S T$ images of ULIRGs are intimately connected with the outflows in these systems. This idea is explored further in $\S 4.1$.

If the dusty outflow attenuates the stellar continuum, it should also attenuate the $\mathrm{Na}$ I D emission line flux, as suggested by the strong correlation between the two (Figure 7). It will not, however, affect the emission line equivalent width if it is a screen in front of both the line emission and stars, since it will attenuate both equally. 

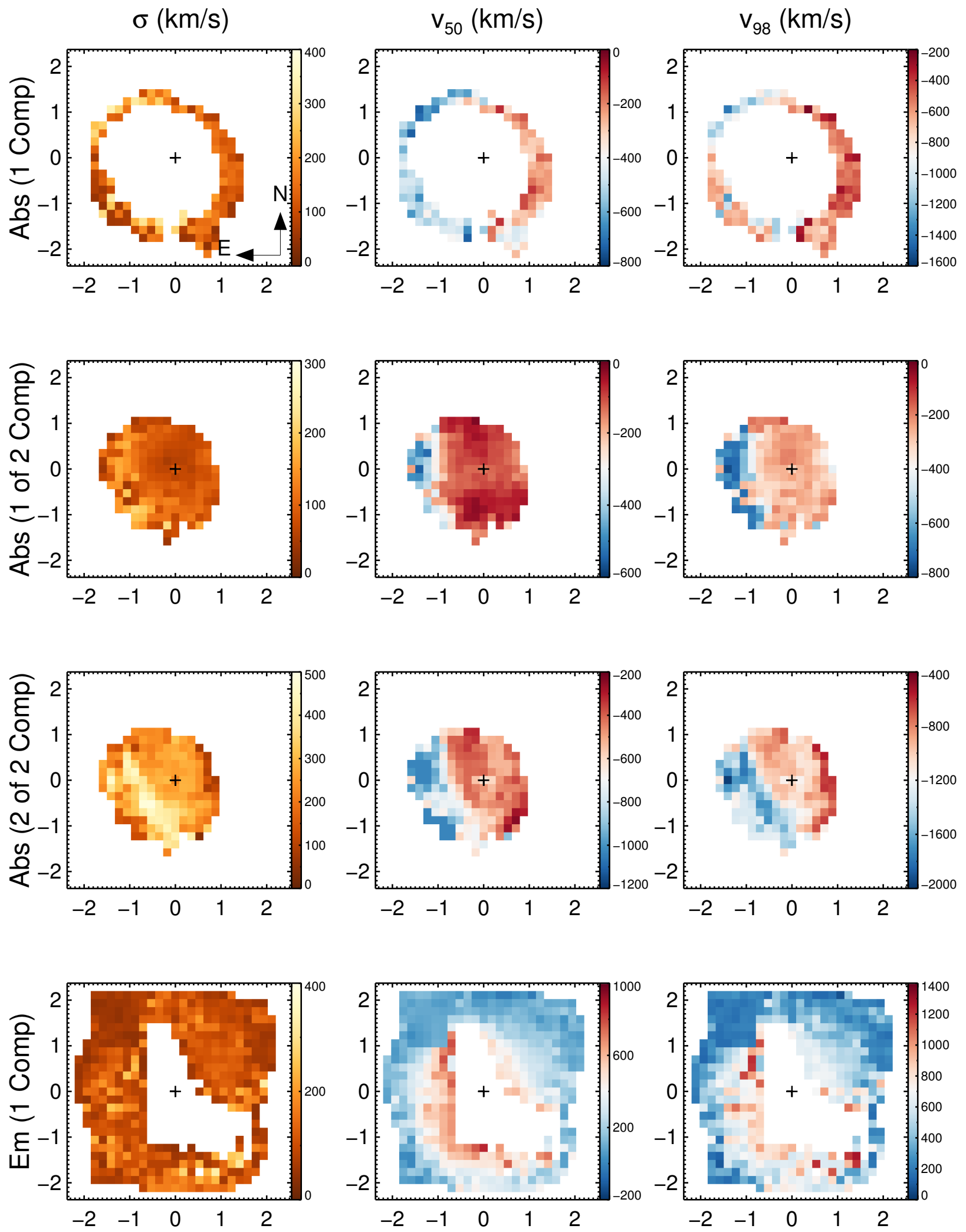

Figure 9. Maps of velocity dispersion (left), $v_{50 \%}$ (middle), and $v_{98 \%} \equiv v_{50 \%} \pm 2 \sigma$ (right) in fits to Na I D. Absorption line fits with one (top) or two (middle) velocity components are shown, as well as emission line fits with one component (bottom). $v_{50 \%}$ and $v_{98} \%$ in the absorption lines peak in a ridge running east to south, $1 \mathrm{kpc}$ in projection from the nucleus, at blueshifted velocities up to $-2000 \mathrm{~km} \mathrm{~s}^{-1}$. The emission line velocities, in contrast, decrease with increasing galactocentric radius $(\S 3.2$. 

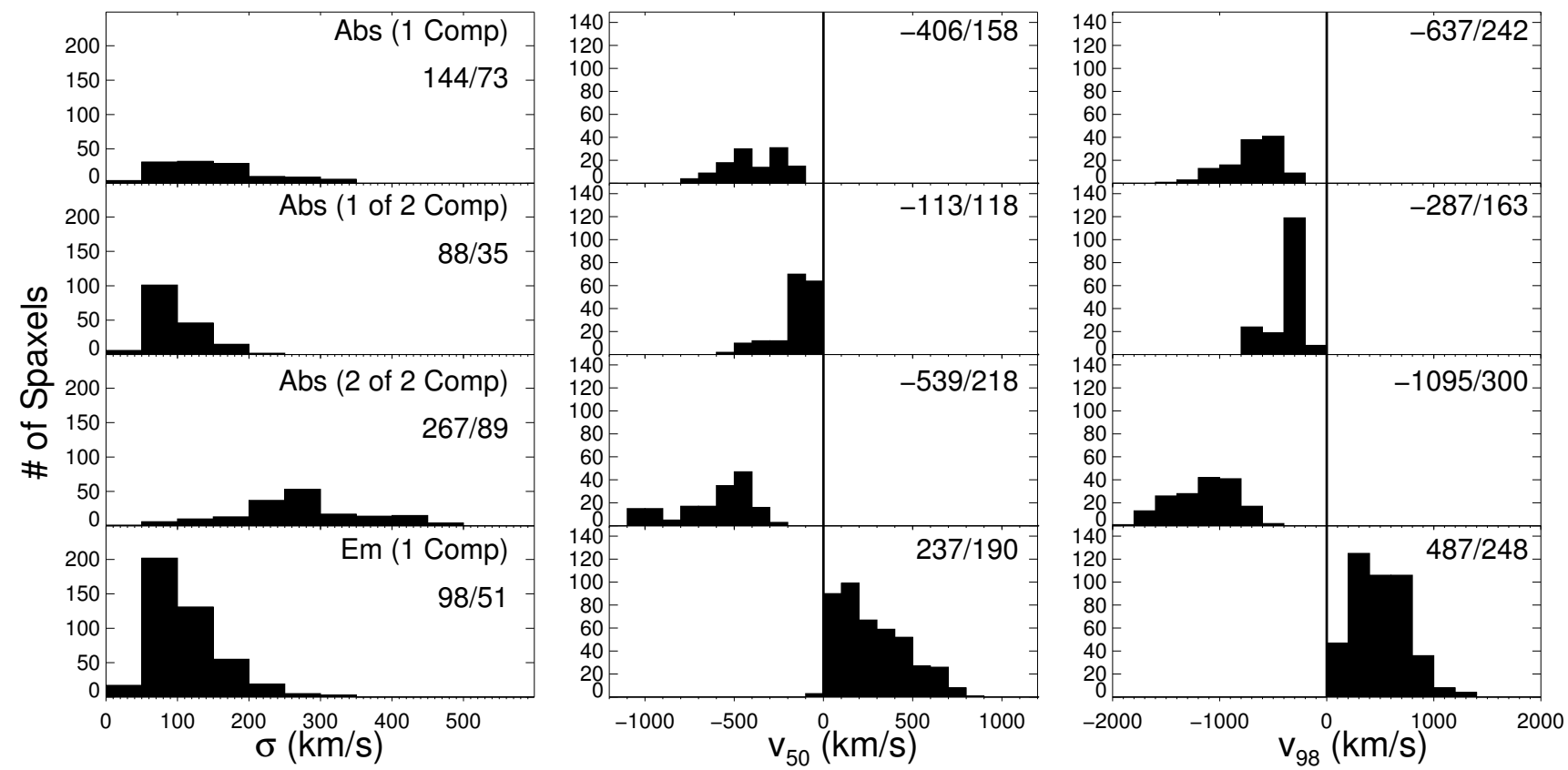

Figure 10. Histograms of velocity dispersion (left), $v_{50 \%}$ (middle), and $v_{98 \%} \equiv v_{50 \%} \pm 2 \sigma$ (right) in fits to Na I D. Absorption lines fits with one (top) or two (middle) components are shown, as well as emission line fits (bottom). The median and standard deviation of each distribution are listed in the upper right corner of each panel. The absolute values of the peak emission line velocities are smaller than those of the absorption lines.

This is consistent with the lack of correlation between $F 435 W-F 814 W$ and $W_{e q}^{e m}$. In $\S 4.1$ a possible model for the Na I D line emission from the wind is presented.

\subsection{Outflow Velocities}

The absorption-line velocities in nuclear spectra of ULIRGs show high blueshifts with a detection rate of $80 \%$, which has been interpreted as evidence for ubiquitous outflowing gas (Heckman et al. 2000, Rupke et al.

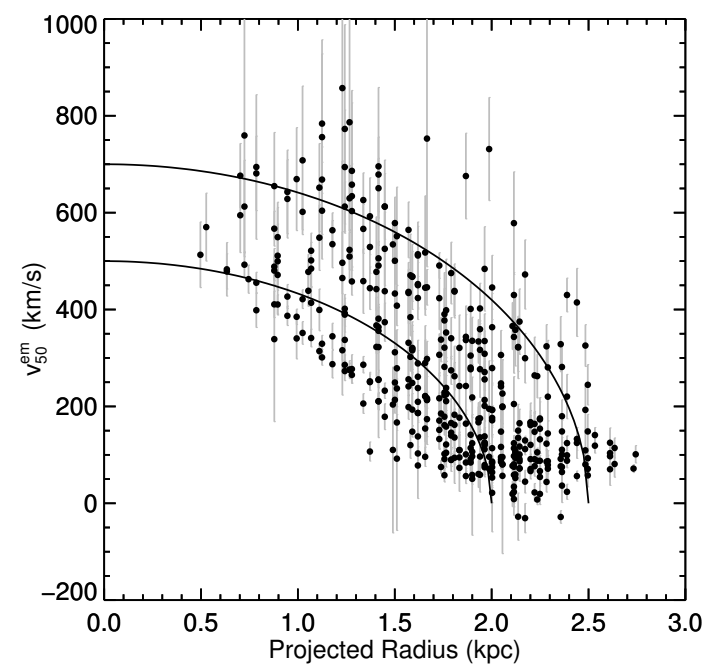

Figure 11. Na I D emission line velocity $\left(v_{50 \%}\right)$ vs. projected galactocentric radius. The lines show projected velocities of thin shells with $v=500 \mathrm{~km} \mathrm{~s}^{-1}, R=2.0 \mathrm{kpc}$ (bottom line) and $v=700$ $\mathrm{km} \mathrm{s}^{-1}, R=2.5 \mathrm{kpc}$ (top line). Though thin shell models are consistent with the data, the dependence of velocity on radius may result in part from emission line filling of absorption lines $(\S 3.2$.
2002, 2005c a; Martin 2005). These neutral outflows have higher velocities in galaxies hosting strong AGN (Rupke \& Veilleux 2013b), an effect that is also seen in the molecular (Veilleux et al. 2013. Cicone et al. 2014) and ionized (Westmoquette et al. 2012, Rupke \& Veilleux 2013b, Arribas et al. 2014) gas phases. Since F05189-2524 is a ULIRG powered by a powerful quasar, high-velocity outflowing gas in the neutral gas phase should be observed. However, the highest projected outflow velocities are typically observed outside of the nuclear line of sight (Rupke \& Veilleux 2013b).

The velocities of resonant line emission due to outflows in ULIRGs are unknown. Observations of two highredshift galaxies with strong, extended $\mathrm{Mg}$ II 2796, 2800 $\AA$ emission both reveal emission lines slightly less offset from systemic than the corresponding absorption lines. Rubin et al. (2011) outline a case with mean absorption velocities of -200 to $-300 \mathrm{~km} \mathrm{~s}^{-1}$ and flux-weighted emission velocities of $30-70 \mathrm{~km} \mathrm{~s}^{-1}$, while Martin et al. (2013) present a galaxy with a velocity of $-230 \mathrm{~km} \mathrm{~s}^{-1}$ in absorption and $110 \mathrm{~km} \mathrm{~s}^{-1}$ in emission (though in this study the absorption line velocity was derived from rest-frame UV Fe II lines). Models predict smaller absolute velocity shifts of emission lines than absorption lines in a given resonant transition (Prochaska et al. 2011). For spherically-symmetric outflow models, the emission line profile is created by multiple absorptions and emissions throughout the nebula, and the integrated emission more closely reflects the average dynamics of the entire outflow. However, it is still redshifted because bluer photons are absorbed by the outflowing regions on the near side of the galaxy (Prochaska et al. 2011).

To parameterize the neutral gas velocities using the fits, each component was considered separately and $v_{50 \%}$ (the center of the analytic profile) and $v_{98 \%}=v_{50 \%} \pm 2 \sigma$ 
(+ for emission, - for absorption) were calculated. The empirical method for constraining Na I D also yields velocity information, and for the absorbing gas this broadly matches the fit results. However, the empirical emission line velocities are noisy due to the low SNR. The fits can also decompose the profile properly into the two doublet components. The empirical measurements do not distinguish between the doublet components except on the profile edges, and thus introduce a velocity uncertainty.

Figures 9 and 10 display maps and histograms of the spatially-resolved velocity distributions in F05189-2524.

The absorption line velocities are everywhere blueshifted, and in both components they peak in a ridge running $1 \mathrm{kpc} \mathrm{E}$ of the nucleus to $1 \mathrm{kpc} \mathrm{S}$ of the nucleus, coincident with the peak in the total equivalent width. The linewidth in the broad component reaches $\sigma=500 \mathrm{~km} \mathrm{~s}^{-1}$, leading to peak velocities of almost $-2000 \mathrm{~km} \mathrm{~s}^{-1}$. These velocities are higher than any measured to date in the neutral phase of an extended outflow in nearby ULIRGs, including Mrk 231 (Rupke et al. 2005c a, Rupke \& Veilleux 2011, 2013b), though ionized gas velocities can exceed this. For spaxels with two fitted components, median values of $v_{50 \%}\left(v_{98 \%}\right)$ are -110 and $-540 \mathrm{~km} \mathrm{~s}^{-1}(-300$ and $\left.-1000 \mathrm{~km} \mathrm{~s}^{-1}\right)$. These values are slightly larger than earlier single-aperture measurements due to the latter being luminosity-weighted. Rupke et al. (2005a) find two absorption line components in the nuclear spectrum of F05189-2524 (through a 1."0 slit), with $v_{50 \%}\left(v_{98 \%}\right)$ values of -90 and $-400 \mathrm{~km} \mathrm{~s}^{-1}(-220$ and $\left.-900 \mathrm{~km} \mathrm{~s}^{-1}\right)$. Teng et al. (2013) find the same velocity components in deep $\mathrm{HI}$ data $\left(v_{50 \%}=-140\right.$ and $-440 \mathrm{~km} \mathrm{~s}^{-1}$, with their data adjusted to correspond to the $z_{\text {sys }}$ used in this paper). The high neutral gas velocities are consistent with those measured in other major mergers containing a quasar (Rupke \& Veilleux 2013b).

The emission line velocities have a strong spatial dependence. The line widths do not vary strongly across the FOV, but the values of $v_{50 \%}$ and $v_{98 \%}$ fall with increasing radius (Figure 11). The highest velocities (exceeding $1000 \mathrm{~km} \mathrm{~s}^{-1}$ ) are not detected at the edges of the FOV, but only within $1-2 \mathrm{kpc}$ of the nucleus. Peak redshifted velocities in the emission lines are smaller than peak blueshifted velocities in the absorption lines, with $v_{98 \%}$ reaching only $1300 \mathrm{~km} \mathrm{~s}^{-1}$ in emission (Figure 10 .

The spatial distribution of emission line $v_{50 \%}$ and $W_{e q}^{e m}$ are very similar. In fact, the two are strongly correlated (Figure 12). In Figure 12, the spaxels are colored according to whether or not they are adjacent to absorption lines (if $\mathrm{Na} \mathrm{I} \mathrm{D}$ absorption is not present in a spaxel) and according to the absorption line equivalent width (if $\mathrm{Na} I \mathrm{D}$ absorption is present in a spaxel). It is apparent that on average, higher $W_{e q}^{a b s}$ corresponds to lower $\left|W_{e q}^{e m}\right|$ and higher emission line velocity. Furthermore, even when absorption is not formally fitted, there is a trend for spaxels with neighboring absorption line fits to have smaller $\left|W_{e q}^{e m}\right|$.

There are two interpretations of these correlations, both of which relate to wind structure (which is discussed further in $\S 4.1$.

First, Figures 11 and 12 could reflect the intrinsic spa-

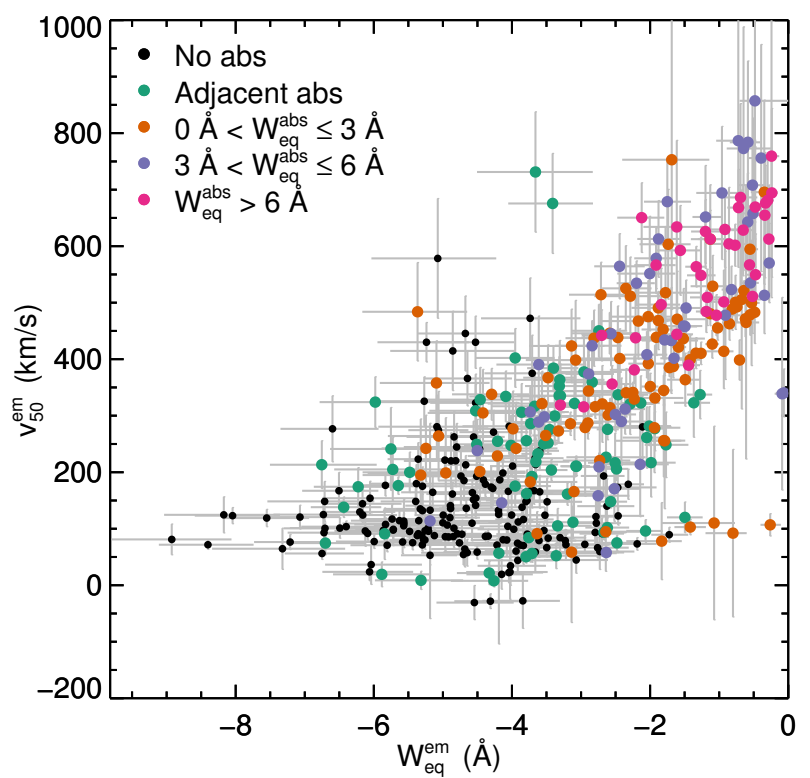

Figure 12. Na I D emission line velocity $\left(v_{50 \%}\right)$ vs. Na I D emission line equivalent width. The two are strongly correlated, and are also correlated with the strength of the Na I D absorption in the same spaxel (as indicated by the colors). Two models that could explain this are (1) a spherically symmetric shell whose projected velocity is higher at smaller radii (Figure 11) or (2) 3D emission-line filling of absorption lines at low equivalent widths, which preferentially fills in velocities near systemic ( $\$ 3.2$ and 4.1 .

tial variation of the projected emission line velocities. If the outflow is an expanding thin shell of gas, then the resonant emission from the far side of the outflow will have a projected velocity that decreases with increasing projected radius. The correlation of velocity with equivalent width in this scenario then implies that $\left|W_{e q}^{e m}\right|$ decreases with increasing projected radius. In Figure 11 two lines show the result of geometric projection of two thin shells, one with $v=500 \mathrm{~km} \mathrm{~s}^{-1}$ and $R=2.0 \mathrm{kpc}$ and the other with $v=700 \mathrm{~km} \mathrm{~s}^{-1}$ and $R=2.5 \mathrm{kpc}$. This range of radii and velocities is consistent with the bulk of absorbing gas on the near side.

A second interpretation is that the increase of redshift with decreasing equivalent width is due to emission line filling of nearby (in wavelength space) absorption, as predicted by Prochaska et al. (2011). In this model, the neutral gas density can vary smoothly with (actual) radius, rather than being concentrated in a thin shell. Filling of the line absorption by emission scattered into the line of sight in a given spaxel simultaneously decreases $W_{e q}^{a b s}$ and $\left|W_{e q}^{e m}\right|$. It should also reduce $I^{e m}$ and increase $v_{50 \%}$, since the line filling of a spherical wind (at least in integrated spectra) occurs preferentially near the systemic velocity (Prochaska et al. 2011). The fact that velocity also correlates with $W_{e q}^{a b s}$ can be explained in this model if the emission line equivalent width is most affected when the absorption is strongest (i.e., there is more absorption to fill in).

\section{DISCUSSION}

The present data are only the second spatially-resolved detection of resonant line emission from cool gas in 
a galactic wind at low redshift (and the first 3D observations at any redshift). These data thus uniquely constrain current models of cool gas in galactic winds (Prochaska et al. 2011), as well as serving as a benchmark for future observations and models.

\subsection{Clues to the Wind Structure from Resonant Emission and Absorption}

Prochaska et al. (2011) presented the first radiative transfer models of cool gas emission and absorption in simple, spherically-symmetric galactic winds that extend from the nucleus out to large radii. They persuasively argued that both absorption and emission are strong in dust-free spherical winds, that the absorption lines are blueshifted and the emission lines near systemic or redshifted, and that the emission extends over the entire wind area with a relatively shallow but declining radial surface brightness profile.

This is broadly consistent with our data. In particular, we observe strong, blueshifted absorption and redshifted emission, as well as emission that extends across the FOV. However, the emission is strongly suppressed in the nuclear regions.

To further explore an emission line model that fills the FOV, Figures 13 and 14 compare the emission line data to a model assuming azimuthal symmetry in the plane of the sky. The model uses a Sérsic surface brightness profile for the integrated $\mathrm{Na}$ I D emission, $I(R)=I_{e} e^{-\left(R / R_{e}\right)^{(1 / n)}}$. This profile was extincted by a foreground screen whose magnitude is inferred from the stellar continuum colors and convolved with the seeing. Only points with low absorption-line contamination, to minimize emission line filling, were fit. The attenuation curve assumed was Calzetti et al. (2000) with $R_{V}=4.85$ (the curve closest to the data in Figure 8), though the result is not at all sensitive to $R_{V}$. The background continuum for computing $W_{e q}$ was the average observed optical continuum in the $50 \AA$ above and below Na I D. A best-fit model with $I_{e}=2.1 \times 10^{-15} \mathrm{erg} \mathrm{s}^{-1} \mathrm{~cm}^{-2}$ $\operatorname{arcsec}^{-2}, R_{e}=2.5 \mathrm{kpc}$, and $n=1.0$ reproduces the ridge of enhanced emission $\mathrm{W}$ of the nucleus, the general features of the equivalent width map, and the correlation of continuum colors with $I^{e m}$. This is similar to the $R$ and $H$-band stellar surface brightness profiles, which have $n=0.8-1$ and $R_{e}=3.9-4.3$ (Veilleux et al. 2002 2006).

At the lowest values of $I^{e m}$ and $W_{e q}^{e m}$, including the central region where only empirical upper (lower) limits exist for $I^{e m}\left(W_{e q}^{e m}\right)$ and Na I D absorption is the strongest, the model begins to deviate from the data (bottom panels of Figure 14. This range of $W_{e q}^{e m}$ also corresponds to the largest deviations of $v_{50 \%}$ from systemic (Figure 12). It is likely that in these regions the emission and absorption lines are being affected by emission-line filling of the absorption lines (Prochaska et al. 2011). Besides reducing $W_{e q}^{e m}$ and increasing $v_{50 \%}$ for the emission lines, this effect will also reduce $W_{e q}^{a b s}$ in these regions by up to $1-2 \AA$, and may push the absorption line solution towards lower optical depths since the filling will preferentially affect the lower optical depth $\mathrm{Na} I \mathrm{D}$ line, $5896 \AA$. Figure 8 suggests that the latter effect is small for most two component absorption line

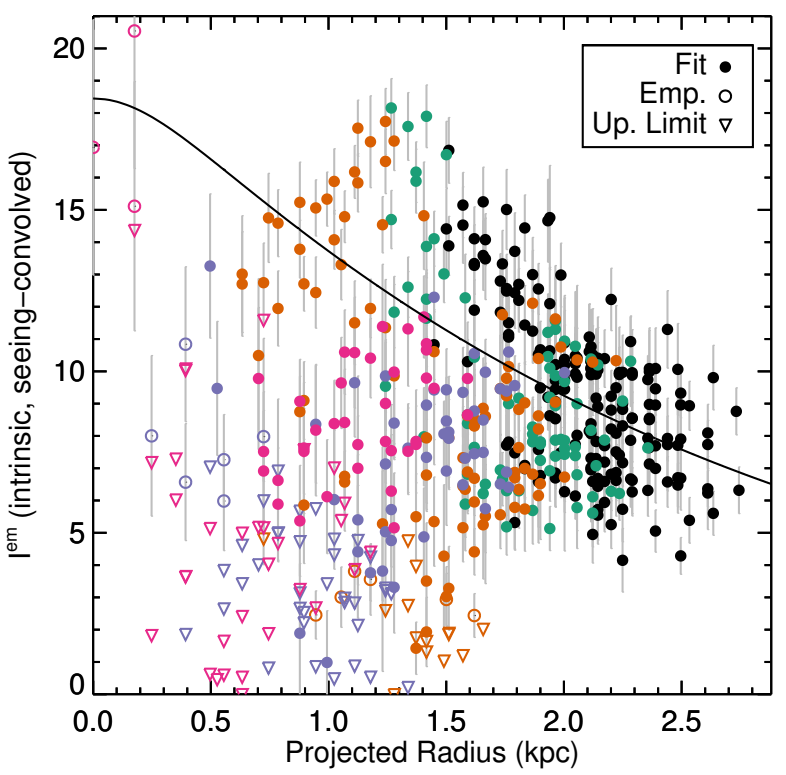

Figure 13. Intrinsic Na I D emission line surface brightness vs. projected radius. Measured values of $I^{e m}$ have been corrected for intrinsic obscuration using continuum colors $(\S 3.1$. The solid points show data from fits to the emission lines, and the open points and triangles show empirical measurements and upper limits in the inner regions where fits are not available. The points are colored according to the contamination from $\mathrm{Na}$ I $\mathrm{D}$ absorption (see Figure 12 for colors). The line is the best fit model to the data, which is a Sérsic profile with $R_{e}=2.5 \mathrm{kpc}$ and $n=1.0$. We fit only points with emission line fits and $W_{e q}^{a b s}<3 \AA$ to minimize contamination from emission line filling $(\S 4.1$ and Figure 14 .

fits, but could impact the low SNR, one component fits.

However, emission line filling is not the only factor responsible for the projected radial velocity profile of the emission line gas (Figure 11). It is apparent that the maximum redshifts $\left(v_{98 \%}\right)$ in the emission lines decrease away from the nucleus (Figure 9), implying that projection effects are also reducing the measured velocities.

This $2 \mathrm{D}$ view of the wind does not directly constrain whether the wind is configured as a thin shell or rather as a radially-continuous, volume-filling sphere. At face value, a radially thin shell in emission would be limbbrightened, contrary to the data, while a radially-filling wind would have the qualitative surface brightness behavior that is observed (Prochaska et al.|2011). However, radiative transfer effects can impact the naive expectation, and intermediate cases such as a thicker shell are also plausible. Further detailed modeling is required before quantitative surface brightness profiles like that discussed here can be used to constrain the density profile of the wind.

It is evident from this analysis that resonant line emission and absorption are complementary probes of galactic outflows. The Na I D absorption in this system traces the dusty outflowing gas $\left(A_{V}=1-4, N(H) \sim 10^{21-22}\right)$ that happens to lie along the line of sight to the galaxy nucleus and its stellar disk. The Na I D emission, conversely, is almost invisible along these same lines of sight because of dust obscuration. Along unobscured lines of sight, emission provides a view of the rest of the wind. This latter view is complicated by radiative 

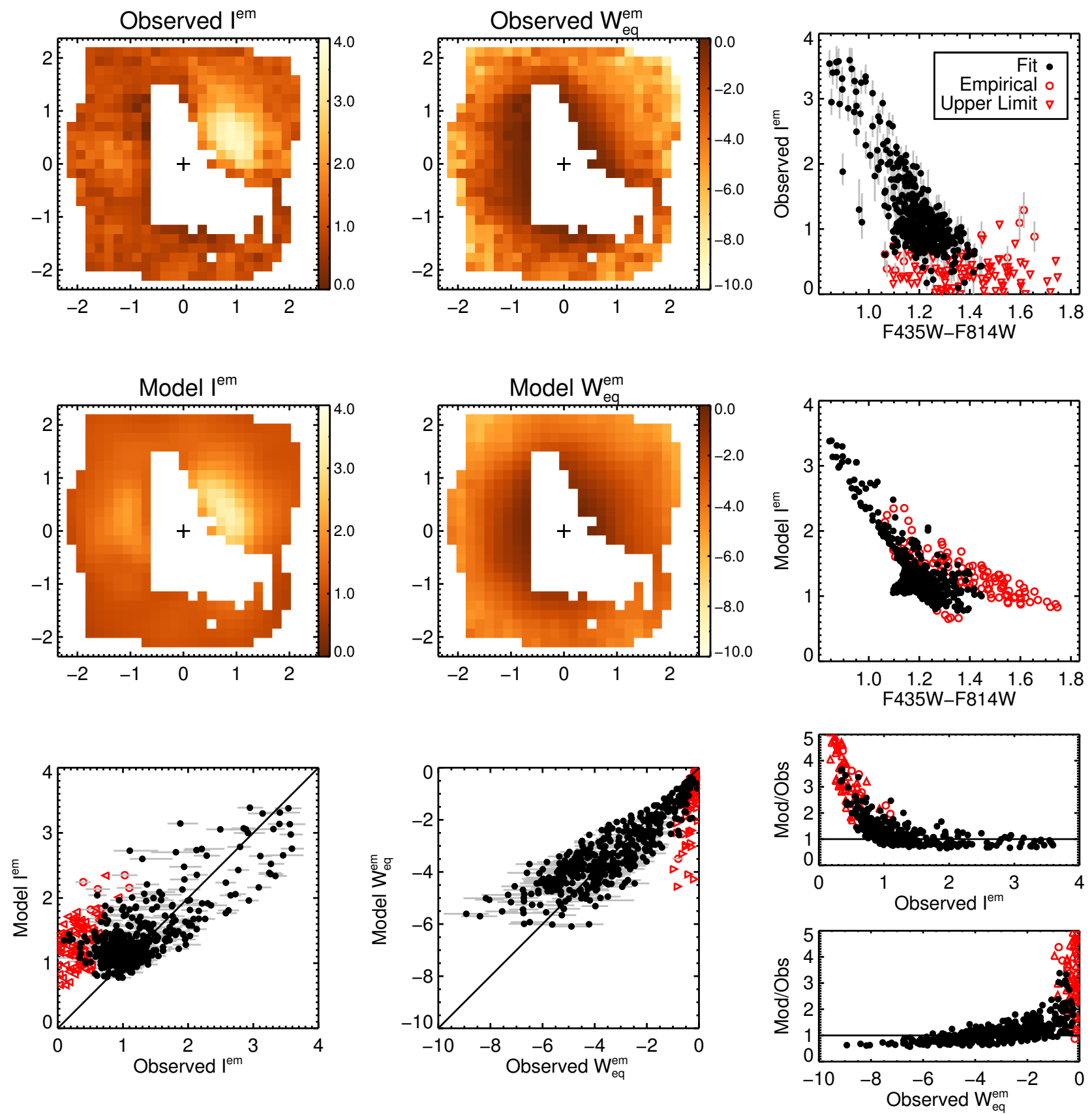

Figure 14. Comparison between observed emission line surface brightnesses, equivalent widths, and continuum colors (top row) and those predicted by the dust-extincted model (middle row). The maps in the middle row show model spaxels for which there is a fitted emission line. The far right middle panel shows these same model points as black circles, while model points which correspond to spaxels in which only empirical estimates or upper limits exist are shown as red open circles. The bottom row quantitatively compares the observed and model values for $I^{\text {em }}$ and $W_{e q}^{e m}$. The best fit model is a Sérsic profile with $n=1.0, I_{e}=2.1 \times 10^{-15} \mathrm{erg} \mathrm{s}^{-1} \mathrm{~cm}^{-2} \operatorname{arcsec}^{-2}$, and a scale length $R_{e}=2.5 \mathrm{kpc}$. This profile is extincted by a foreground screen whose magnitude is inferred from the stellar continuum colors and convolved with the seeing. There is excellent overall correspondence between the two (bottom row) except at the lowest values of $I^{e m}$ and $W_{e q}^{e m}$ (bottom right). At these levels, the spaxels are primarily in the inner $\mathrm{kpc}$ in regions of strong absorption and no emission line fit; these spaxels are represented in the above plots by empirical estimates and upper limits. Some emission line filling of the absorption lines, which reduces both $I^{e m}$ and $W_{e q}^{e m}$, may explain the discrepancy $(\$ 4.1$. 
transfer effects which lower the measured velocity if the wind is volume-filling rather than configured as a shell (Prochaska et al. 2011).

The presence of Na I D emission across the FOV suggests a wind that is more extended than one would infer from $\mathrm{Na} I \mathrm{D}$ absorption alone (and vice versa, since the two peak in strength in very different places). Absorption line probes show that the neutral phase of the outflows can extend to $10 \mathrm{kpc}$ or farther in ULIRGs (Martin 2006). The wind in this system extends to at least the edge of the FOV $(3 \mathrm{kpc})$, and is apparently optically thick to these radii in integrated line emission $(\S 2$. Deep longslit observations may constrain the point at which the wind becomes optically thin, and thus the true size of the wind. This probe will be more sensitive to the true extent than absorption lines, since no background continuum is required and the equivalent width is increasing with increasing radius (Figure 5). The two high- $z$ examples also show a more extended outflow in emission than absorption (Rubin et al. 2011; Martin et al. 2013).

The collimation of the F05189-2524 outflow is consistent with that of other ULIRG winds, though the current data do not constrain the inner collimation. Spatiallyresolved studies of neutral, ionized, and molecular gas outflows in ULIRGs reveal that the gas preferentially emerges along the minor axis of the nuclear disk at radii of $1-2 \mathrm{kpc}$, but becomes less collimated at larger radii (Rupke et al. 2005c; Rupke \& Veilleux 2013b a). If an observer could choose a random nuclear line of sight through a given wind, they would detect an outflow in absorption $\sim 80 \%$ of the time (Rupke et al. 2005c). The absorption line map of this galaxy is consistent with this result. Although we detect nuclear absorption, our observations trace only one nuclear sightline through the wind. A clumpy and/or collimated wind could be less detectable through other nuclear sightlines. The data are consistent with a collimated inner wind if the nuclear disk is near face-on or an uncollimated wind if the nuclear disk is closer to edge-on (there is no published data on the nuclear disk in this galaxy). Our model for the observed $\mathrm{Na}$ I D emission is symmetric in the plane of the sky, but since it is detected only at scales $\gtrsim 0.5 \mathrm{kpc}$ this is consistent with either an uncollimated wind or the decreasing collimation with increasing radius in other ULIRGs outflows (Rupke \& Veilleux 2013b).

These results also show that foreground dust is one of the primary drivers of the observed morphology of Na I D emission and absorption in this galaxy. Na atoms ionize easily (the first ionization potential is $5.1 \mathrm{eV}$ ), so the spatial correspondence between absorbing $\mathrm{Na}$ atoms and dust is natural, since dust can shield the atoms from ionizing radiation. The observed peak in Na I D flux (Figure 5] is deceptive, in that it more closely reflects the foreground screen than the actual distribution of emission line gas (which in the model is azimuthally symmetric).

\subsection{Detection of Extended $\mathrm{NaI} D$ Emission}

As is evident from many long-slit studies (Heckman et al. 2000; Rupke et al. 2002, 2005b c, Martin 2005), high surface brightness resonant line emission is not a common feature of galactic winds in nearby galaxies. Prochaska et al. (2011) list dust, wind asymmetry, and emissionline filling of absorption lines as possible physical causes of these non-detections.

$\mathrm{Na}$ I D line emission is in fact present at low levels in stacked spectra of face-on galaxies with low dust attenuation (Chen et al. 2010), suggesting that both dust and asymmetry could indeed play a role in affecting detectability of $\mathrm{Na}$ I D emission. At high $z$, Kornei et al. (2013) found that $A_{U V}$ is at least partly responsible for the varying strength of $\mathrm{Mg}$ II absorption and Fe II* emission in galactic winds. The equivalent width and/or detection rate of $\mathrm{Mg}$ II emission in high $z$ star forming galaxies is also higher in galaxies with bluer UV colors (Weiner et al. 2009, Erb et al. 2012, Kornei et al. 2013).

The current data show that dust is pivotal in shaping the morphologies of both $\mathrm{Na}$ I D absorption and emission. However, it does not obviously affect the detectability of $\mathrm{Na}$ I D emission. The $A_{V}$ values in F05189-2524 are typical for a ULIRG (Surace et al. 1998, Veilleux et al. 1999a). The highest emission line flux is indeed in the region of lowest extinction and absent in the regions of highest extinction, but is detectable throughout the FOV, up to $3 \mathrm{kpc}$ from the galaxy nucleus. As for asymmetry, the 3D structure of the outflow in F05189-2524 is not constrained beyond the surface brightness emission model, but the data do not point to any unusual asymmetry that would affect the resonant lines differently than in other systems.

The two high $z$ galaxies with extended Mg II emission have not only blue UV colors, but also high restframe blue luminosity compared to similar galaxies $(\mathrm{Ru}-$ bin et al. 2011; Martin et al. 2013). A necessary physical condition for strong resonant lines is enough continuum emission at the same wavelength as the transition to excite the atoms out of the ground state. Higher $R$-band luminosity could thus in principle strengthen Na I D emission.

In fact, within a nuclear aperture of $4 \mathrm{kpc}$, F05189-2524 has $M_{R, 4 \mathrm{kpc}}=-20.44$, which is brighter than $90 \%$ of ULIRGs in the 1 Jy sample (Kim et al. 2002) and is negligibly affected by the nuclear point source (Kim et al.2013). Furthermore, most of the galaxies that are brighter than F05189-2524 are Seyfert 1s, in which the light is concentrated in an unresolved point source and possibly beamed preferentially along the line of sight to Earth. Excluding the Seyfert 1s in the parent sample of ULIRGs, F05189-2524 is brighter than 95\% of the parent sample at $R$, within a $4 \mathrm{kpc}$ nuclear aperture. Of the five non-Seyfert-1 ULIRGs that have lower $M_{R, 4 \mathrm{kpc}}$ than F05189-2524, four have been previously observed with long-slit spectra (Rupke et al. 2005b c a) and one (Mrk 273) also with IFS (Rupke \& Veilleux 2013b). None show obvious Na I D emission.

This suggests that the nuclear $R$-band luminosity of F05189-2524 is responsible for the detection of extended $\mathrm{Na}$ I D emission, though it may not in fact be a sufficient condition for its detection. However, deeper IFS observations of other dusty, $R$-luminous systems will be necessary to confirm this. In long-slit observations, slit loss could be also a factor if the line emission is spatially extended at a low surface brightness (Prochaska et al. 2011), though that is clearly not a factor in the current data or in other IFS studies of Na I D Rupke \& Veilleux 2013b). Nonetheless, it could be responsible for the lack of observed $\mathrm{Na}$ I D emission in long slit data (Rupke et al. 2005b c a; Martin 2005). Furthermore, purely nu- 
clear spectra in these dusty systems are less likely to show Na I D emission, since the nucleus is typically the most heavily obscured part of the galaxy and will be dominated by absorption rather than emission.

Several other nearby galaxies show resonant emission from metal lines, but there is presently nothing to distinguish their luminosities. NGC 1808 is fainter at $R$ (in terms of integrated brightness) than other nearby AGN (Koss et al. 2011), with $M_{R} \sim-21$ (NED). In a nearUV study of five $z \sim 0.25$ ULIRGs, Martin \& Bouché (2009) find $\mathrm{Mg}$ II emission in two Seyfert 2s, but in neither case is this emission extended. The relevant continuum luminosity for this transition at these redshifts is observed-frame $U$-band. No $U$ measurements exist for this sample, though integrated $U$-band luminosities in nearby ULIRGs do not reveal significant differences between Seyfert 2s and non-Seyfert spectral types (Surace \& Sanders 2000).

In other respects, however, the $\mathrm{Na}$ I D absorption and emission in NGC 1808 are similar to that described here. NGC 1808 has a nuclear obcuration similar to F05189-2524 $\left(A_{V}=3-4\right.$; Kewley et al. 2001). Its outflowing dust streamers, which are prominent in optical color maps $(B-R)$, also show Na I D absorption. However, these streamers are less obscured than much of the Na I D absorbing region of F05189-2524 $\left(A_{V}<1\right.$; Phillips 1993). Finally, the emission on the far side of NGC 1808 emerges in a relatively unobscured region; they appear just outside dust lanes in the galaxy disk.

Should Na I D emission be detectable outside the GMOS FOV? The emission line model for F05189-2524 has an exponential scale length of $2.5 \mathrm{kpc}$, and spaxels near the field edge (at $1-2$ scale lengths) still have $I^{e m} \sim 10^{-16} \mathrm{erg} \mathrm{s}^{-1} \mathrm{~cm}^{-2} \operatorname{arcsec}^{-2}$. Prochaska et al. (2011) predict that line emission extends to a radius where the optical depth still exceeds a few tenths, while the Na I D line emission in the GMOS FOV is optically thick. Furthermore, if the wind is isotropic, the total absorption line and emission line equivalent widths of an integrated spectrum should be equal (Prochaska et al. 2011). However, summing the GMOS spectra across the FOV yields $W_{e q}^{a b s}=3.1 \AA$ and $W_{e q}^{e m}=-0.7 \AA$. If the wind in F05189-2524 is fairly isotropic, a significant amount of emission (in terms of equivalent width) lies outside the GMOS FOV. A deep long-slit spectrum or narrow-band image should be able to constrain the extent of the $\mathrm{Na} I \mathrm{D}$ emission in this system.

How would F05189-2524 look at a greater distance? Almost all observations of resonant line emission have been made at $z \gtrsim 0.5$ with rest-frame UV lines. At these redshifts, $\mathrm{Na}$ I D is in the observed-frame near-infrared, a wavelength range in which sensitive absorption line measurements of individual objects are challenging due to sky emission and absorption lines and lower CCD sensitivity. Furthermore, in this system the primary source of Poisson noise is not from the emission lines but rather the continuum.

Despite these difficulties, detection of $\mathrm{Na}$ I D emisison in an object similar to F05189-2524 is reasonable at $z \lesssim 0.5$ if strong sky lines are avoided. For instance, at $z=0.3$ a 1 .'0 slit covers the GMOS FOV. Summing the GMOS spectra across the FOV in the present data shows that emission is detected with $W_{e q}^{e m}=-0.7 \AA$ and a flux of $1.5 \times 10^{-15} \mathrm{erg} \mathrm{s}^{-1} \mathrm{~cm}^{-2}$. Cosmological surface brightness dimming at $z \sim 0.3$ amounts to a factor of three. The Gemini/GMOS Integration Time Calculator indicates that less than an hour of spectroscopic integration in a $1^{\prime \prime} \times 1^{\prime \prime}$ aperture yields a signal-to-noise ratio in which the emission can be easily distinguished from the continuum (the emission line flux is $\sim 10 \times$ the noise from the continuum). Even at $z=0.5$, the continuum signal-to-noise ratio in one hour is high enough that the emission line flux is several times larger than the noise in the continuum. At higher redshifts, the detectability drops sharply due to sky lines and CCD sensitivity.

\section{SUMMARY}

We present the second example of resolved $\mathrm{Na} \mathrm{I} \mathrm{D}$ emission from a galactic wind in the nearby universe. The GMOS IFU follows Na I D absorption and emission across a $4 \times 4 \mathrm{kpc}$ aperture in a nearby ULIRG hosting a quasar. These resolved observations provide unique constraints on models and future observations of resonant line emission in galactic winds.

The Na I D absorption lines trace the dusty near side of the wind. F05189-2524 shows the highest velocity kpcscale neutral gas outflow to be directly resolved in any nearby galaxy, with blueshifted velocities up to almost $-2000 \mathrm{~km} \mathrm{~s}^{-1}$. The present IFS data directly connect the absorption in the outflow with the foreground dust column obscuring the stellar continuum. Fits to the absorption lines with low covering factors yield optically thick absorption with $N(H) \sim 10^{21-22} \mathrm{~cm}^{-2}$. The column density is correlated with the dust column in a way that matches the column density expected if the wind has a Galactic dust-to-gas ratio.

The optically thick Na I D emission lines extend to the edges of the FOV, but are extincted in the inner regions of the galaxy. An azimuthally symmetric Sérsic model, with the emission extincted by the same foreground screen as the stellar continuum, reproduces the observed emission line surface brightness and equivalent width maps at all but the lowest values of $I^{e m}$ and $\left|W_{e q}^{e m}\right|$. At these levels, which correspond to the inner regions of the wind nearest the regions of $\mathrm{Na} I \mathrm{D}$ absorption, emission line filling of the $\mathrm{Na}$ I D absorption lines near the systemic velocity is a likely culprit, as suggested by correlations of $W_{e q}^{e m}$ with emission line velocity and $W_{e q}^{a b s}$.

The morphology of the resonant line absorption and emission in this galaxy are clearly regulated by the presence of dust. Furthermore, the detection of extended $\mathrm{Na}$ I D emission in this system in particular may be due to its unusually high continuum surface brightness at the rest wavelength of the resonant transition, similar to galaxies with extended $\mathrm{Mg}$ II emission observed in the rest-frame UV at high $z$ (Rubin et al. 2011, Martin et al. 2013). Future observations of Na I D in other nearby or low redshift systems $(z \sim 0-0.5)$ with high rest-frame $R$ band surface brightness may be able to detect extended $\mathrm{Na}$ I D emission due to galactic winds.

This unique dataset shows that $\mathrm{Na}$ I D absorption and emission are very complementary probes of wind structure. $W_{e q}^{a b s}$ and $W_{e q}^{e m}$ are anti-correlated on a spaxel-byspaxel basis, and the $I^{e m}$ and $W_{e q}^{a b s}$ peaks are on opposite sides of the nucleus. The absorption lines trace the near side of the wind along the line of sight to Earth, while the 
emission lines trace the bulk of the interior and far side of the wind through radiative transfer (Prochaska et al. 2011). Together, they form a picture of a dusty, wide angle wind that extends to at least several kpc from the nucleus in most directions. This picture is consistent with previous work on galactic winds in major mergers (Rupke et al. 2005c; Martin 2006, Rupke \& Veilleux 2013b).

We thank the referee for their thorough and helpful comments. This work was based on observations obtained at the Gemini Observatory (program ID GS2011B-Q-64), which is operated by the Association of Universities for Research in Astronomy, Inc., under a cooperative agreement with the NSF on behalf of the Gemini partnership: the National Science Foundation (United States), the Science and Technology Facilities Council (United Kingdom), the National Research Council (Canada), CONICYT (Chile), the Australian Research Council (Australia), Ministério da Ciência, Tecnologia e Inovação (Brazil) and Ministerio de Ciencia, Tecnología e Innovación Productiva (Argentina). D.S.N.R. was supported by a Cottrell College Science Award from the Research Corporation for Science Advancement and by NASA grant Keck/JPL RSA 1461849. S.V. was supported in part by NSF grant AST1009583 and NASA grants NHSC/JPL RSA 1427277 and 1454738.

The HST observations described here were obtained from the Hubble Legacy Archive, which is a collaboration between the Space Telescope Science Institute (STScI/NASA), the Space Telescope European Coordinating Facility (ST-ECF/ESA) and the Canadian Astronomy Data Centre (CADC/NRC/CSA).

\section{REFERENCES}

Allington-Smith, J., et al. 2002, PASP, 114, 892

Armus, L., et al. 2007, ApJ, 656, 148

-. 2009, PASP, 121, 559

Arribas, S., Colina, L., Bellocchi, E., Maiolino, R., \& Villar-Martín, M. 2014, A\&A, 568, A14

Bellocchi, E., Arribas, S., Colina, L., \& Miralles-Caballero, D. 2013, A\&A, 557, A59

Calzetti, D. 2001, PASP, 113, 1449

Calzetti, D., Armus, L., Bohlin, R. C., Kinney, A. L., Koornneef, J., \& Storchi-Bergmann, T. 2000, ApJ, 533, 682

Cappellari, M., \& Emsellem, E. 2004, PASP, 116, 138

Chen, Y.-M., Tremonti, C. A., Heckman, T. M., Kauffmann, G., Weiner, B. J., Brinchmann, J., \& Wang, J. 2010, AJ, 140, 445 Cicone, C., et al. 2014, A\&A, 562, A21

Cooksey, K. L., Prochaska, J. X., Chen, H.-W., Mulchaey, J. S., \& Weiner, B. J. 2008, ApJ, 676, 262

Erb, D. K., Quider, A. M., Henry, A. L., \& Martin, C. L. 2012, ApJ, 759, 26

Farrah, D., Surace, J. A., Veilleux, S., Sanders, D. B., \& Vacca, W. D. 2005, ApJ, 626, 70

Feruglio, C., Maiolino, R., Piconcelli, E., Menci, N., Aussel, H., Lamastra, A., \& Fiore, F. 2010, A\&A, 518, L155

González Delgado, R. M., Cerviño, M., Martins, L. P., Leitherer, C., \& Hauschildt, P. H. 2005, MNRAS, 357, 945

Heckman, T. M., Lehnert, M. D., Strickland, D. K., \& Armus, L. 2000, ApJS, 129, 493

Hobbs, L. M. 1974, ApJ, 191, 381
Hook, I. M., Jørgensen, I., Allington-Smith, J. R., Davies, R. L., Metcalfe, N., Murowinski, R. G., \& Crampton, D. 2004, PASP, 116,425

Kewley, L. J., Heisler, C. A., Dopita, M. A., \& Lumsden, S. 2001, ApJS, 132, 37

Kim, D.-C., Veilleux, S., \& Sanders, D. B. 2002, ApJS, 143, 277

Kim, D.-C., et al. 2013, ApJ, 768, 102

Kornei, K. A., Shapley, A. E., Martin, C. L., Coil, A. L., Lotz, J. M., \& Weiner, B. J. 2013, ApJ, 774, 50

Koss, M., Mushotzky, R., Veilleux, S., Winter, L. M., Baumgartner, W., Tueller, J., Gehrels, N., \& Valencic, L. 2011, ApJ, 739, 57

Leitherer, C., et al. 1999, ApJS, 123, 3

Lupton, R. H., Gunn, J. E., \& Szalay, A. S. 1999, AJ, 118, 1406

Martin, C. L. 2005, ApJ, 621, 227

—. 2006, ApJ, 647, 222

Martin, C. L., \& Bouché, N. 2009, ApJ, 703, 1394

Martin, C. L., Shapley, A. E., Coil, A. L., Kornei, K. A., Bundy, K., Weiner, B. J., Noeske, K. G., \& Schiminovich, D. 2012, ApJ, 760, 127

Martin, C. L., Shapley, A. E., Coil, A. L., Kornei, K. A., Murray, N., \& Pancoast, A. 2013, ApJ, 770, 41

Munari, U., \& Zwitter, T. 1997, A\&A, 318, 269

Phillips, A. C. 1993, AJ, 105, 486

Predehl, P., \& Schmitt, J. H. M. M. 1995, A\&A, 293, 889

Prochaska, J. X., Kasen, D., \& Rubin, K. 2011, ApJ, 734, 24

Rodríguez Zaurín, J., Tadhunter, C. N., \& González Delgado, R. M. 2009, MNRAS, 400, 1139

-. 2010, MNRAS, 403, 1317

Rubin, K. H. R., Prochaska, J. X., Ménard, B., Murray, N., Kasen, D., Koo, D. C., \& Phillips, A. C. 2011, ApJ, 728, 55

Rubin, K. H. R., Weiner, B. J., Koo, D. C., Martin, C. L., Prochaska, J. X., Coil, A. L., \& Newman, J. A. 2010, ApJ, 719, 1503

Rupke, D. S., Veilleux, S., \& Sanders, D. B. 2002, ApJ, 570, 588

-. 2005a, ApJ, 632, 751

-. 2005b, ApJS, 160, 87

-. 2005c, ApJS, 160, 115

Rupke, D. S. N. 2014a, IFSFIT: Spectral Fitting for Integral Field Spectrographs, Astrophysics Source Code Library

—. 2014b, IFSRED: Data Reduction for Integral Field

Spectrographs, Astrophysics Source Code Library

Rupke, D. S. N., \& Veilleux, S. 2011, ApJ, 729, L27

-. 2013a, ApJ, 775, L15

-. 2013b, ApJ, 768, 75

Rupke, D. S. N., Veilleux, S., \& Baker, A. J. 2008, ApJ, 674, 172

Sanders, D. B., Scoville, N. Z., \& Soifer, B. T. 1991, ApJ, 370, 158

Shapley, A. E., Steidel, C. C., Pettini, M., \& Adelberger, K. L. 2003, ApJ, 588, 65

Shih, H., \& Rupke, D. S. N. 2010, ApJ, 724, 1430

Soto, K. T., \& Martin, C. L. 2010, ApJ, 716, 332

Sturm, E., et al. 2011, ApJ, 733, L16

Surace, J. A., \& Sanders, D. B. 2000, AJ, 120, 604

Surace, J. A., Sanders, D. B., Vacca, W. D., Veilleux, S., \& Mazzarella, J. M. 1998, ApJ, 492, 116

Teng, S. H., Veilleux, S., \& Baker, A. J. 2013, ApJ, 765, 95

U, V., et al. 2012, ApJS, 203, 9

Veilleux, S., Cecil, G., \& Bland-Hawthorn, J. 2005, ARA\&A, 43, 769

Veilleux, S., Kim, D.-C., \& Sanders, D. B. 1999a, ApJ, 522, 113

—. 2002, ApJS, 143, 315

Veilleux, S., Kim, D.-C., Sanders, D. B., Mazzarella, J. M., \& Soifer, B. T. 1995, ApJS, 98, 171

Veilleux, S., Sanders, D. B., \& Kim, D.-C. 1999b, ApJ, 522, 139

Veilleux, S., et al. 2006, ApJ, 643, 707

-. 2009, ApJS, 182, 628

-. 2013, ApJ, 776, 27

Vergely, J.-L., Valette, B., Lallement, R., \& Raimond, S. 2010, A\&A, 518, A31

Weiner, B. J., et al. 2009, ApJ, 692, 187

Westmoquette, M. S., Clements, D. L., Bendo, G. J., \& Khan, S. A. 2012, MNRAS, 3173 